\title{
Axial anomaly in multi-Weyl and triple-point semimetals
}

\author{
Luca Lepori, $^{a, b}$ Michele Burrello $^{c}$ and Enore Guadagnini ${ }^{d, e}$ \\ ${ }^{a}$ Dipartimento di Scienze Fisiche e Chimiche, Università dell'Aquila, \\ via Vetoio, I-67010 Coppito-L'Aquila, Italy \\ ${ }^{b}$ INFN, Laboratori Nazionali del Gran Sasso, \\ Via G. Acitelli 22, I-67100 Assergi (AQ), Italy \\ ${ }^{c}$ Niels Bohr International Academy and Center for Quantum Devices, University of Copenhagen, \\ Juliane Maries Vej 30, 2100 Copenhagen, Denmark \\ ${ }^{d}$ Dipartimento di Fisica E. Fermi, Università di Pisa, \\ Largo B. Pontecorvo 3, 56127 Pisa, Italy \\ ${ }^{e}$ INFN, Sezione di Pisa, \\ Largo B. Pontecorvo 3, 56127 Pisa, Italy \\ E-mail: llepori81@gmail.com, michele.burrello@gmail.com, \\ enore.guadagnini@unipi.it
}

ABStRaCt: We derive the expression of the abelian axial anomaly in the so-called multiWeyl and triple-point crossing semimetals. No simplifying restrictions are assumed on the symmetry of the spectrum. Three different computation methods are considered: the perturbative quantum field theory procedure which is based on the evaluation of the one-loop Feynman diagrams, the Nielsen-Ninomiya method, and the Atiyah-Singer index argument. It is shown that the functional form of the axial anomaly does not depend on the Lorentz symmetry, but it is determined by the gauge structure group. We discuss the stability of the anomaly — stemming from the quantisation of the anomaly coefficient — under smooth modifications of the lagrangian parameters.

Keywords: Anomalies in Field and String Theories, Chiral Lagrangians, Effective Field Theories, Topological States of Matter

ARXIV EPRINT: 1803.01684 


\section{Contents}

1 Introduction $\quad 2$

2 Basic features of topological semimetals $\quad 4$

$\begin{array}{lll}3 & \text { Overview of the results } & 6\end{array}$

3.1 Anomalous axial symmetry for Weyl fermions 6

$\begin{array}{lll}3.2 & \text { Double-Weyl semimetals } & 7\end{array}$

3.3 Triple-Weyl semimetals 8

3.4 Triple-point semimetals 9

4 Gauge anomalies in perturbative quantum field theory 10

$\begin{array}{ll}4.1 \text { Perturbative approach } & 10\end{array}$

$\begin{array}{ll}4.2 & \text { Anomaly as a solution of a cohomological problem } \\ \end{array}$

$\begin{array}{lll}4.3 & \text { Axial anomaly } & 13\end{array}$

5 Perturbative computation $\quad \mathbf{1 5}$

$\begin{array}{lll}5.1 \text { Regularization } & 15\end{array}$

$\begin{array}{ll}5.2 \text { Gauge variation } & 16\end{array}$

$\begin{array}{lll}5.3 & \text { Computation rules } & 17\end{array}$

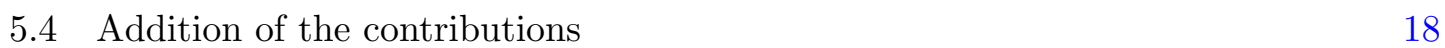

$\begin{array}{ll}\text { 5.5 The final result } & 19\end{array}$

6 Nielsen-Ninomiya procedure $\quad 20$

6.1 Classical external fields 20

6.2 Normalizable zero-energy modes 21

$\begin{array}{lll}6.3 & \text { Dirac sea and the axial anomaly } & 23\end{array}$

7 Atiyah-Singer index $\quad 25$

$\begin{array}{lll}7.1 & \text { Particles and antiparticles } & 26\end{array}$

$\begin{array}{lll}7.2 & \text { Euclidean zero modes } & 26\end{array}$

8 Axial anomaly for triple-Weyl semimetals 28

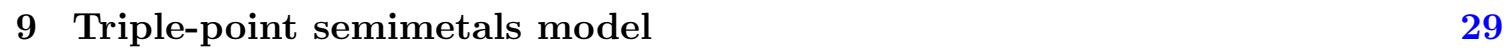

10 Quantization of the anomaly coefficient 31

11 Chemical potentials and stability $\quad 35$

$\begin{array}{ll}11.1 \text { Multi-Weyl semimetals } & 35\end{array}$

$\begin{array}{ll}11.2 \text { Triple-point semimetals } & 37\end{array}$ 


\section{Introduction}

Anomalies are known since long ago in the context of quantum field theory [1-3]. In the construction of a quantum field theory based on a classical lagrangian, it may happen that a certain symmetry of the classical action cannot be preserved at the quantum level; when this happens, the symmetry is called anomalous. A well known example of anomaly is found when chiral fermions are minimally coupled with gauge fields; in this case, some of the symmetries acting on the fermion fields may be broken at the quantum level because of the so-called chiral anomalies [4-8]. In elementary particle physics, the experimental consequences of the flavour chiral anomalies have been described for instance in $[5-7,9]$.

In the last decade it has been realized that the study of anomalies in field theories is also a fundamental tool for the effective description of topological phases of matter such as topological insulators and superconductors [10,11]. In this scenario it has been shown that a field theoretical approach accounting for chiral and gravitational anomalies allows us to characterize the peculiar transport properties of topological materials resulting from the coupling to electromagnetic fields or temperature gradients [12, 13]. In particular, anomalies provide a natural description for phenomena like the surface Hall conductance, which are related to the gapless surface modes of these gapped systems and constitute a useful tool for their classification.

Since the work by Nielsen and Ninomiya [14] it has been known that also gapless models can enjoy similar topological responses under external electromagnetic fields, thus actualizing the effects of quantum anomalies. Only recently, however, similar topological gapless phases of matter have gained a considerable attention and have been experimentally realized in solid state materials [15-18]. The main example is provided by Weyl semimetals [19, 20], which constitute a remarkable embodiment of the Dirac theory for massless fermions. In the presence of magnetic fields, they display transport properties which are dictated by the corresponding chiral anomaly [13] and have been studied in recent works [21-25]. These three dimensional systems host pairs of inequivalent and isolated Weyl points (or Weyl nodes) in the Brillouin zone. These are points where two energy bands touch each-other, with a linear dispersion that determines the appearance of a cone. The Weyl nodes appear always in pairs for lattice models [26] and can be separated in momentum space by breaking the space inversion or time reversal canonical symmetries [19, 20, 27]. In their neighborhood, thus for energies close to the band touching points, the fermionic quasiparticles display a linear dispersion law and their dynamics can be effectively described by a Weyl hamiltonian involving a pair of cones with opposite "chirality". The appearance of Weyl points in pairs has a topological origin [26, 28]; moreover it also implies (if spacerotational symmetry is present close to the nodes) the emergence of an effective Lorentz covariance, characterizing the low-energy dynamics of the Weyl semimetal, the chemical potential is assumed to coincide with the energy of the Weyl nodes. 
Weyl semimetals present indeed non-trivial topological features, exactly due to the Weyl nodes which constitute a monopole source for the Berry connection of the bands [29]. This topology manifests in the presence of gapless chiral modes, exponentially localized on the surfaces of these systems. At the energy of the Weyl nodes, such surface states identify lines in the momentum space connecting the projections on the surface Brillouin zone of two inequivalent nodes; these surface modes are therefore dubbed Fermi arcs [19]. Weyl semimetals and Fermi arcs have been realized and detected in various compounds, including for instance tantalum arsenide [15-17], niobium arsenide [18], bismuth trisodium [30], and tantalum phosphide [31]. Their implementation, however, was not limited to solid-state materials only, but it also encompasses other platforms, for example in photonic [32-34] and phononic [35] crystals.

Quite recently, Weyl semimetals (which we will dub single-Weyl semimetals in the following, to avoid confusion) have been shown to admit notable generalizations in the so-called multi-Weyl semimetals [36], lattice systems displaying isolated band touching points, similar to the Weyl nodes, but where the dispersion law is linear along one space direction only and grows with a higher power of the momenta along the other two directions. Double-Weyl nodes have been predicted in a number of rare-earth compounds [37-42], in ultracold-atoms set-ups [43-45] and in photonic crystals [46].

The dispersion relation of the multi-Weyl points implies a breaking of the effective (lowenergy) Lorentz covariance, which characterizes instead the single-Weyl cones. Despite the absence of the Lorentz symmetry, it has been argued [47-49] that, for particular values of the lagrangian parameters, the axial anomaly assumes the standard functional form that one derives in Lorentz invariant theories. The anomalous Hall conductivity which characterizes multi-Weyl materials does not depend indeed on the effective Lorentz covariance of the system, which is always violated when one takes into account the band dispersion. The latter property has been exemplified by even more exotic topological semimetals, characterized by the simultaneous merging of multiple energy bands, such that they can be interpreted as models with a modified spin-statistics relationship [50]. A notable example involves triplepoint semimetals [51-53], recently realized [54] in molybdenum phosphide, where three bands cross in a triply-degenerate point with a multiple topological charge. A summary of some of the main features of these topological semimetals is provided in section 2 .

The main scope of the present work is the computation of the abelian anomalies related to suitable global transformations of anticommuting fields, which appear in the lagrangian models describing double-Weyl, triple-Weyl and triple-point lattice systems around the nodal points. These anomalies are connected with the so-called axial transformations acting on the fermion fields in the presence of an abelian vector potential $A_{\mu}(x)$ (see [49] and references therein). An overview of our results is presented in section 3 .

No simplifying restrictions are assumed on the symmetry of the spectrum of the various models. The considered low energy lagrangians are not Lorentz invariant; indeed, the relevant differential operators acting on the fermionic variables are not necessarily described by homogeneous polynomials of the covariant derivatives, and may contain dimensioned parameters in front of them. Let us recall that the standard results [4-8] concerning the axial anomaly have been obtained in the presence of Lorentz invariance. So the computation 
of the axial anomaly in the case of models which are not Lorentz invariant presents original aspects. Therefore, in order to make our article self-contained, in sections 4-7 we describe in detail our anomaly computations in the case of the double-Weyl semimetal model; the triple-Weyl and triple-point models are examined subsequently.

Three different methods for the derivation of the anomaly are considered: the perturbative quantum field theory procedure [4-8] which is based on the evaluation of the one-loop Feynman diagrams, the Nielsen-Ninomiya method [14], and the Atiyah-Singer index argument $[55,56]$. The mutual consistency of these methods is illustrated. The general features of the perturbative approach are described in section 4, where the relationship between the chiral anomaly and the axial anomaly is produced. The perturbative computations of the chiral anomaly for the double-Weyl model are contained in section 5, the Nielsen-Ninomiya procedure is presented in section 6, and the anomaly derivation by means of the AtiyahSinger index argument is contained in section 7. The axial anomaly for the triple-Weyl and the triple-point models are derived in section- 8 and section 9 respectively. In these cases, the perturbative approach is rather arduous or affected by singularities; therefore only the Nielsen-Ninomiya and Atiyah-Singer methods are considered. The quantization of the multiplicative anomaly coefficient is discussed in section 10, which also contains a few comments on the structure of the obtained anomaly expressions, as well as on their stability under smooth perturbations of the lagrangian parameters. The effects on the axial anomaly of modifications of the chemical potentials are examined in detail in section 11, where we also illustrate certain peculiar features of the anomaly computation in the case of the triple-point model. Finally, our conclusions are presented in section 12.

\section{Basic features of topological semimetals}

The prediction and discovery of symmetry-protected topological matter is considered one of the crucial achievements of the theory of condensed matter in the last decades. The most prominent example in this set is given by topological insulators [10, 11]. These are gapped materials, non-interacting or weakly interacting, which present gapless modes localized on their surface or edges and responsible for their transport properties. These surface modes, in general, maintain their gapless and localized nature as long as certain discrete symmetries of the system are preserved or a phase transition through a critical point of the bulk occurs. Topological insulators can be efficiently described in terms of non-interacting lattice models and the existence of their gapless edge or surface modes can be deduced, in general, by topological indices, such as Chern or winding numbers, characterizing their energy bands in the Brillouin zone of the lattice.

The study of topological features in condensed matter materials extended rapidly to gapless systems (see, for example, the reviews [57, 58]), namely topological metals and semimetals. A semimetal is a material that presents two partially filled energy bands at its chemical potential; this implies that there are at least two energy bands overlapping in energy. The limiting case is provided by energy bands with a discrete set of band touching points in the Brillouin zone and the chemical potential lying exactly at the energy of these points. The most typical example of topological semimetal is the Weyl semimetal that 
(a)

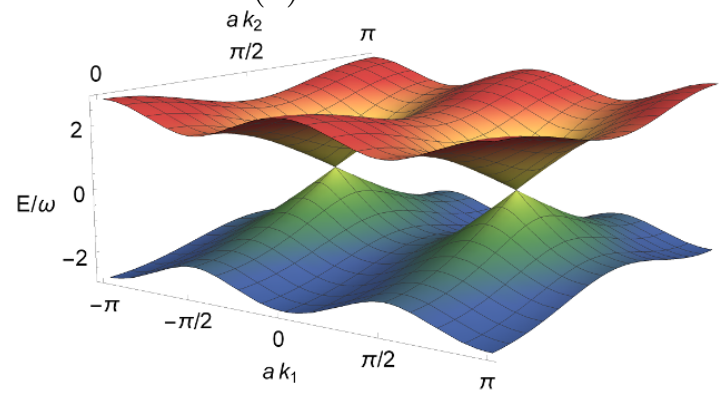

$(c)$

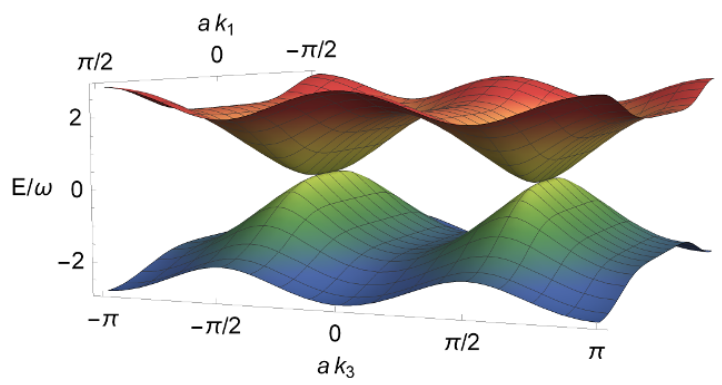

(b)

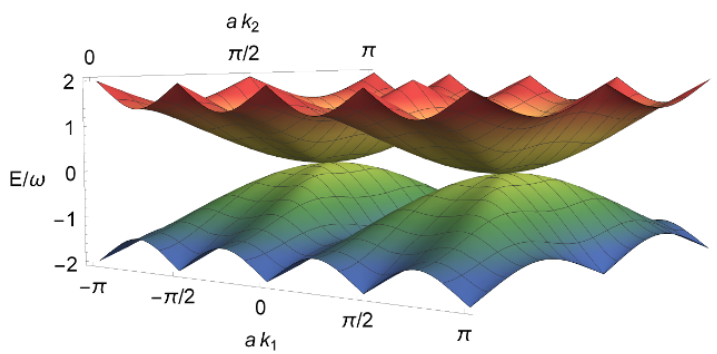

$(d)$

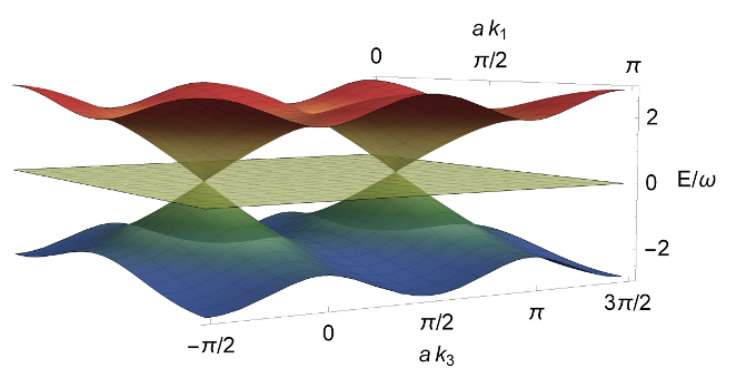

Figure 1. Typical dispersions of tight-binding lattice models corresponding to the classes of topological semimetals considered in this paper. $\omega$ is an arbitrary energy scale and a labels the lattice spacing. (a) Dispersion as a function of $k_{1}$ and $k_{2}$ of a single-Weyl semimetal corresponding to the model in [27]: it displays pairs of linearly dispersing Weyl cones with opposite chirality in its Brillouin zone. (b) Spectrum of a double-Weyl semimetal from the lattice model in [43]: the energy is plotted as a function of $k_{1}$ and $k_{2}$, which are the directions with quadratic dispersion. The value of $k_{3}$, along which the dispersion is linear, has been fixed at the band touching points. Around the double-Weyl points, the system displays a $C_{4}$ rotational symmetry with axis along the $k_{3}$ direction. (c) Typical spectrum of a triple-Weyl semimetal as a function of $k_{1}$ and $k_{3}$. The dispersion is cubic in $k_{1}$ (and $k_{2}$, not shown) and linear in $k_{3} . k_{2}$ has been chosen at the band touching point. $(d)$ Dispersion of the triple-point semimetal model in [59]. The triple point crossings are band touching points connecting a flat band at zero-energy and two linearly dispersing bands.

belongs indeed to this case: a Weyl semimetal is a three-dimensional material with two bands touching with a linear dispersion along all the directions in an even number of points in the Brillouin zone (see figure 1(a)). These gapless points are robust against any small translational-invariant perturbation of the system, as long as they lie at different momenta of the Brillouin zone. Any pair of these nodes with opposite chiralities can be efficiently described in terms of two decoupled Weyl hamiltonians; therefore, for energies close to the band-touching points (thus small temperatures and chemical potentials close to the Weyl points) these systems are characterized by an emerging Lorentz invariance; increasing or decreasing the chemical potential, instead, the non-trivial dispersion of the bands become relevant to determine the physical properties of the system.

The linear-dispersing single-Weyl points correspond to unitary monopoles of the Berry curvature calculated on the two touching energy bands [29]. These points do not exhaust 
the possible topological nodes among different bands: in the presence of additional symmetries (e.g. rotational crystal symmetries) it is possible to engineer materials displaying multi-Weyl points [36], which correspond to higher monopoles of the Berry curvature, at the price of giving away the emergent Lorentz invariance. In particular, double-Weyl and triple-Weyl points are stabilized in physical material by the discrete rotational symmetries $C_{4}$ and $C_{6}$ and, in general, they do not display a full rotational $\mathrm{SO}(2)$ symmetry. DoubleWeyl points are characterized by a quadratic dispersion along two directions $\left(k_{1}\right.$ and $k_{2}$ in figure $1(b))$ and by a linear dispersion in the third direction. Triple-Weyl points, instead, display a cubic dispersion along two directions and a linear dispersion along the third one (in figure $1(c)$ an example with linear dispersion along $k_{3}$ and cubic dispersion along $k_{1}$ is displayed). The triple-Weyl case is the one with the highest power in the dispersion law allowed by point group symmetries [36] in spatial dimensions equal or lower than three; by including multi-component fermions with additional symmetries, however, it is possible to engineer band-touching points with even larger dispersion powers and monopole charges.

Finally it is also possible to create systems where three bands connect together in a topological node with double monopole charge [50]. This is the case of the triple-point semimetals with a typical dispersion depicted in figure $1(d)$. If time-reversal symmetry is preserved, the upper and lower bands display a linear dispersion, whereas the central band is flat (it may display as well a quadratic dispersion along all directions, but the gradient of the energy with momentum vanishes in the triple-point crossing). When time-reversal symmetry is broken, also the central band may acquire a dispersion [59].

In the following, our analysis of the axial anomalies which characterize these models is based on lagrangian descriptions of these systems, for energies close to the band-touching points, where the lattice effect can be considered negligible.

\section{Overview of the results}

Our results are summarized in this section. In order to fix the notation, it is useful to add a brief introductory note.

\subsection{Anomalous axial symmetry for Weyl fermions}

The concept of chiral anomaly developed originally in quantum field theory [4-6]. Let us consider for instance the model of massless electrodynamics with lagrangian

$$
\mathcal{L}=\bar{\psi}(x)\left[i \gamma^{\mu} D_{\mu}\right] \psi(x)=\psi_{R}^{\dagger}(x)\left[i D_{0}+i \boldsymbol{\sigma} \cdot \mathbf{D}\right] \psi_{R}(x)+\psi_{L}^{\dagger}(x)\left[i D_{0}-i \boldsymbol{\sigma} \cdot \mathbf{D}\right] \psi_{L}(x),
$$

where $D_{\mu}=\partial_{\mu}+i A_{\mu}(x)$ represents the covariant derivative, $x \equiv x^{\mu}=\left(x^{0}, x^{1}, x^{2}, x^{3}\right), \sigma^{i}$ (with $i=1,2,3$ ) denote the Pauli matrices, and $\gamma^{\mu}$ (with $\mu=0,1,2,3$ ) the Dirac matrices, here in the Weyl representation. The lagrangian (3.1) is invariant under local vector $\mathrm{U}(1)_{V}$ gauge transformations

$$
e^{i \Lambda(x)} \in \mathrm{U}(1)_{V}, \quad\left\{\begin{array}{l}
\psi_{R}(x) \longrightarrow \psi_{R}^{\Lambda}(x)=e^{i \Lambda(x)} \psi_{R}(x) \\
\psi_{L}(x) \longrightarrow \psi_{L}^{\Lambda}(x)=e^{i \Lambda(x)} \psi_{L}(x) \\
A_{\mu}(x) \longrightarrow A_{\mu}^{\Lambda}(x)=A_{\mu}(x)-\partial_{\mu} \Lambda(x)
\end{array},\right.
$$


and under global axial $\mathrm{U}(1)_{A}$ transformations

$$
e^{i \phi} \in \mathrm{U}(1)_{A}, \quad\left\{\begin{array}{l}
\psi_{R}(x) \longrightarrow \psi_{R}^{\phi}(x)=e^{i \phi} \psi_{R}(x) \\
\psi_{L}(x) \longrightarrow \psi_{L}^{\phi}(x)=e^{-i \phi} \psi_{L}(x) \\
A_{\mu}(x) \longrightarrow A_{\mu}(x)
\end{array}\right.
$$

At the classical level, the Noether axial current $J_{A}^{\mu}$ corresponding to the symmetry (3.3) satisfies $\partial_{\mu} J_{A}^{\mu}=0$. However, at the quantum level the axial current is not conserved because of the so-called axial anomaly. When the vector gauge symmetry (3.2) is preserved, the axial anomaly can be written in the form [4-6]

$$
\partial_{\mu} J_{A}^{\mu}(x)=-\frac{1}{4 \pi^{2}} \epsilon^{\mu \nu \tau \lambda} \partial_{\mu} A_{\nu}(x) \partial_{\tau} A_{\lambda}(x)=-\frac{1}{4 \pi^{2}} F^{* \tau \lambda}(x) F_{\tau \lambda}(x),
$$

where $F_{\tau \lambda}(x)=\partial_{\tau} A_{\lambda}(x)-\partial_{\lambda} A_{\tau}(x)$ denotes the electromagnetic tensor, and $F^{* \tau \lambda}(x)=$ $\frac{1}{4} \epsilon^{\mu \nu \tau \lambda} F_{\mu \nu}(x)$.

The same result is known to be also valid for the single-Weyl semimetals [13, 14], where the theory (3.1) describes the low-energy dynamics around the nodal points; there the Pauli matrices act on appropriate "chiral indices", generally labelling the sublattices that form the Weyl semimetal.

\subsection{Double-Weyl semimetals}

Double-Weyl semimetals contain two inequivalent band touching points, protected by a symmetry $C_{4}$, with a linear dispersion relation along the direction connecting them, and a quadratic dispersion relation along the other two directions [36]. In real space-time, the corresponding low energy lagrangian for the fermionic variables associated with these points takes the form $(\hbar=c=1)$

$$
\begin{aligned}
\mathcal{L}= & \psi_{R}^{\dagger}(x)\left[i D_{0}-\alpha\left(D_{1}^{2}-D_{2}^{2}\right) \sigma^{1}-\frac{1}{2} \beta\left\{D_{1}, D_{2}\right\} \sigma^{2}+i \gamma D_{3} \sigma^{3}\right] \psi_{R}(x) \\
& +\psi_{L}^{\dagger}(x)\left[i D_{0}-\alpha\left(D_{1}^{2}-D_{2}^{2}\right) \sigma^{1}-\frac{1}{2} \beta\left\{D_{1}, D_{2}\right\} \sigma^{2}-i \gamma D_{3} \sigma^{3}\right] \psi_{L}(x),
\end{aligned}
$$

where $\alpha, \beta$ and $\gamma$ denote three nonvanishing real constants (in the following, $\gamma$ and $\gamma^{\mu}$ should not be confused). The fermionic (anticommuting) fields $\psi_{R}(x)$ and $\psi_{L}(x)$ have two components but, differently form the case of massless electrodynamics, they do not represent Lorentz spinor fields. The variables $\psi_{R}(x)$ and $\psi_{L}(x)$ are associated to opposite monopole charges, \pm 2 , for the Berry flux around the corresponding nodes. We call them "right" and "left" variables just for notational simplicity. In terms of the four components field $\Psi(x)=\left(\psi_{R}(x), \psi_{L}(x)\right)$, the lagrangian (3.5) can also be written as

$$
\mathcal{L}=\bar{\Psi}(x)\left\{i \gamma_{0} D_{0}-\alpha \gamma_{1} \gamma_{5}\left[D_{1}^{2}-D_{2}^{2}\right]-(\beta / 2) \gamma_{2} \gamma_{5}\left\{D_{1}, D_{2}\right\}+i \gamma \gamma_{3} D_{3}\right\} \Psi(x) .
$$

This expression makes it clear that, in this model, one has a violation of the "canonical" form of the PT-symmetry, which is required to obtain stable multi-Weyl nodes [19]-[27]. 
The lagrangian (3.5) is invariant under the local vector $\mathrm{U}(1)_{V}$ gauge transformations shown in equation (3.2), and under global axial $\mathrm{U}(1)_{A}$ transformations of equation (3.3). The Noether axial current $J_{A}^{\mu}(x)$ corresponding to the symmetry (3.3) can be written as

$$
J_{A}^{\mu}(x)=J_{R}^{\mu}(x)-J_{L}^{\mu}(x),
$$

where

$$
\begin{aligned}
J_{R}^{0} & =\psi_{R}^{\dagger} \psi_{R} & J_{L}^{0} & =\psi_{L}^{\dagger} \psi_{L} \\
J_{R}^{1} & =i \psi_{R}^{\dagger}\left[\alpha \sigma^{1} \overleftrightarrow{D}_{1}+\frac{1}{2} \beta \sigma^{2} \overleftrightarrow{D}_{2}\right] \psi_{R} & J_{L}^{1} & =i \psi_{L}^{\dagger}\left[\alpha \sigma^{1} \overleftrightarrow{D}_{1}+\frac{1}{2} \beta \sigma^{2} \overleftrightarrow{D}_{2}\right] \psi_{L} \\
J_{R}^{2} & =i \psi_{R}^{\dagger}\left[-\alpha \sigma^{1} \overleftrightarrow{D}_{2}+\frac{1}{2} \beta \sigma^{2} \overleftrightarrow{D}_{1}\right] \psi_{R} & J_{L}^{2} & =i \psi_{L}^{\dagger}\left[-\alpha \sigma^{1} \overleftrightarrow{D}_{2}+\frac{1}{2} \beta \sigma^{2} \overleftrightarrow{D}_{1}\right] \psi_{L} \\
J_{R}^{3} & =\gamma \psi_{R}^{\dagger} \sigma^{3} \psi_{R} & J_{L}^{3} & =-\gamma \psi_{L}^{\dagger} \sigma^{3} \psi_{L}
\end{aligned}
$$

in which we have introduced the notation $\psi^{\dagger} \overleftrightarrow{D}_{j} \psi \equiv \psi^{\dagger}\left(D_{j} \psi\right)-\left(D_{j} \psi^{\dagger}\right) \psi$.

In this article we show that, for arbitrary nonvanishing values of $\alpha, \beta$ and $\gamma$, when the gauge invariance (3.2) is maintained the axial anomaly is given by

$$
\partial_{\mu} J_{A}^{\mu}(x)=-2 \Theta(\alpha, \beta, \gamma) \frac{1}{4 \pi^{2}} \epsilon^{\mu \nu \tau \lambda} \partial_{\mu} A_{\nu}(x) \partial_{\tau} A_{\lambda}(x),
$$

where

$$
\Theta(\alpha, \beta, \gamma)=\frac{\alpha \beta \gamma}{|\alpha \beta \gamma|}
$$

For fixed values of $\alpha, \beta$ and $\gamma$, expression (3.9) is twice the chiral anomaly (3.4) that one finds in massless electrodynamics. In section 4 and section 5 , we demonstrate equation (3.9) by means of a one-loop calculation based on Feynman diagrams. Then the same result is rederived by means of the Nielsen-Ninomiya procedure in section 6 , and by means of the Atiyah-Singer index argument in section 7.

The expression for the axial anomaly in equation (3.9) makes its Lorentz independence explicit. The Minkowski metric tensor $\eta_{\mu \nu}$ does not appear in equation (3.9) while the tensor $\epsilon^{\mu \nu \tau \lambda}$ has a "cohomological" origin - as described in section 4 - in view of the fact that the anomaly can be described by a differential 4 -form.

\subsection{Triple-Weyl semimetals}

Triple-Weyl semimetals contain two inequivalent band touching points, protected by a symmetry $C_{6}$, with a linear dispersion relation along the direction connecting them and a cubic dispersion relation along the two remaining directions [36]. The corresponding low energy lagrangian can be written in real space-time as

$$
\begin{aligned}
& \mathcal{L}=\psi_{R}^{\dagger}(x)\left[i D_{0}-\alpha\left(D_{1}^{3}-\mathcal{S}\left[D_{2}^{2}, D_{1}\right]\right) \sigma^{1}-\beta\left(D_{2}^{3}-\mathcal{S}\left[D_{1}^{2}, D_{2}\right]\right) \sigma^{2}+i \gamma D_{3} \sigma^{3}\right] \psi_{R}(x)+ \\
& +\psi_{L}^{\dagger}(x)\left[i D_{0}-\alpha\left(D_{1}^{3}-\mathcal{S}\left[D_{2}^{2}, D_{1}\right]\right) \sigma^{1}-\beta\left(D_{2}^{3}-\mathcal{S}\left[D_{1}^{2}, D_{2}\right]\right) \sigma^{2}-i \gamma D_{3} \sigma^{3}\right] \psi_{L}(x),
\end{aligned}
$$

where the symbol $\mathcal{S}\left[P^{2}, Q\right] \equiv P Q P+P P Q+Q P P$ implements the correct symmetrization of the covariant derivatives, and $\alpha, \beta$ and $\gamma$ denote three nonvanishing real constants. 
In this case also $\psi_{R}(x)$ and $\psi_{L}(x)$ represent two-components anticommuting fields. Precisely like the case of the double-Weyl semimetals, the lagrangian (3.11) is invariant under local vector $\mathrm{U}(1)_{V}$ gauge transformations (3.2) and under global axial $\mathrm{U}(1)_{A}$ transformations (3.3). By means of the Nielsen-Ninomiya procedure and the Atiyah-Singer index argument, in section 8 we shown that, when the gauge invariance (3.2) is maintained, the axial anomaly is given by

$$
\partial_{\mu} J_{A}^{\mu}(x)=-3 \Theta(\alpha, \beta, \gamma) \frac{1}{4 \pi^{2}} \epsilon^{\mu \nu \tau \lambda} \partial_{\mu} A_{\nu}(x) \partial_{\tau} A_{\lambda}(x)
$$

The results in equations (3.4), (3.9) and (3.12) have been first inferred in [60] through a semiclassical calculation based on the kinetic theory of Landau Fermi liquids, and in [48] by means of numerical and analytical approaches. In [48], the authors analyzed numerically the case with parallel electric and magnetic fields, and presented analytical calculations in the spirit of Nielsen and Ninomyia [14] for a system in which both fields are aligned along the direction of linear dispersion and in the presence of a full $\mathrm{SO}(2)$ rotational symmetry. Finally, equation (3.9) has also been verified in [49] through a field theoretical approach, based on the Fujikawa's method (see for example [56, 61]), by evaluating the chiral anomaly for a double-Weyl point with $\mathrm{SO}(2)$ rotational symmetry. In all these papers, in the expression of the anomaly — which has been derived in these articles - the $\Theta(\alpha, \beta, \gamma)$ factor (3.10) is missing. But the absolute value of the anomaly, which has been proposed in $[48,49,60]$, appears to be correct. The difference between the two derivations is that, in $[48,49,60]$, the left field has been considered set since the beginning by the condition $\alpha \beta \gamma>0$.

The derivations in $[48,49]$ do not fully explain the quantization of the anomaly, with topological charge $\mathcal{N} \Theta(\alpha, \beta, \gamma)$, and, from a more fundamental point of view, do not clarify why the anomaly for multi-Weyl semimetals is proportional to the differential form $F(x) \wedge F(x)$, in spite of the breaking of Lorentz covariance of the corresponding low energy lagrangians. In facts, doubts $[62,63]$ have been cast upon the use of the regularization scheme of the path-integral measure exploited in the Fujikawa's method [49] in cases different from the standard Weyl theory.

\subsection{Triple-point semimetals}

Triple-point semimetals [50] are characterized by two zero-energy points in which three bands join. The lagrangian of the low energy model takes the form

$$
\begin{aligned}
\mathcal{L}= & \psi_{R}^{\dagger}(x) i\left[D_{0}-v M_{1} D_{1}-v M_{2} D_{2}-v M_{3} D_{3}\right] \psi_{R}(x) \\
& +\psi_{L}^{\dagger}(x) i\left[D_{0}-v M_{1} D_{1}-v M_{2} D_{2}+v M_{3} D_{3}\right] \psi_{L}(x)
\end{aligned}
$$


where the real parameter $v$ is positive and $D_{\mu}=\partial_{\mu}+i A_{\mu}(x)$. The fermionic fields $\psi_{R}(x)$ and $\psi_{L}(x)$ have three components. The three matrices $M_{j}$ are given by

$$
\begin{aligned}
& M_{1}=M_{1}(\theta)=\left(\begin{array}{ccc}
0 & 0 & 0 \\
0 & 0 & i e^{i \theta} \\
0 & -i e^{-i \theta} & 0
\end{array}\right), \quad M_{2}=M_{2}(\theta)=\left(\begin{array}{ccc}
0 & 0 & i e^{-i \theta} \\
0 & 0 & 0 \\
-i e^{i \theta} & 0 & 0
\end{array}\right), \\
& M_{3}=M_{3}(\theta)=\left(\begin{array}{ccc}
0 & i e^{i \theta} & 0 \\
-i e^{-i \theta} & 0 & 0 \\
0 & 0 & 0
\end{array}\right),
\end{aligned}
$$

in which the angle $\theta$ is a parameter of the model which breaks time-reversal symmetry. The $M_{j}$ matrices can be interpreted ad deformed generators of the rotation group in the adjoint representation; in our notation these matrices satisfy the commutation relations:

$$
\left[M_{j}\left(\theta_{1}\right), M_{k}\left(\theta_{2}\right)\right]=i \epsilon_{j k \ell} M_{\ell}\left(-\theta_{1}-\theta_{2}\right) .
$$

The lagrangian (3.13) is invariant under local vector gauge transformations (3.2) and under global axial transformations (3.3). When the vector gauge invariance is preserved, the axial anomaly is found to be

$$
\partial_{\mu} J_{A}^{\mu}(x)=\frac{\cos (3 \theta)}{|\cos (3 \theta)|} \frac{1}{4 \pi^{2}} \epsilon^{\mu \nu \tau \lambda} \partial_{\mu} A_{\nu}(x) \partial_{\tau} A_{\lambda}(x) .
$$

This expression is derived in section 9 by means of the Nielsen-Ninomiya and Atiyah-Singer methods.

\section{Gauge anomalies in perturbative quantum field theory}

Before proceeding with the direct computation of the anomaly, it is useful to discuss the relationship between the so-called left-right and vector-axial possible forms of the anomaly, together with a few general properties of gauge anomalies.

\subsection{Perturbative approach}

In order to simplify the exposition and avoid repetitions, in the following discussion we concentrate directly on the double-Weyl model (3.5); but the results of this section clearly have a general validity. It is convenient to examine first the lagrangian terms for the massless fermionic fields $\psi_{R}(x)$ and $\psi_{L}(x)$ separately; afterwards, the anomalous behaviours of their corresponding one-loop diagrams will be combined in order to determine the desired axial anomaly. Let us consider the "right-handed" component $\psi_{R}(x)$. In order to simplify the exposition, in the intermediate steps of the computation the gauge field coupled with $\psi_{R}(x)$ will be denoted by $V_{\mu}(x)$. We shall recover the previous $A_{\mu}(x)$ notation at the end of the present section. The corresponding lagrangian density $\mathcal{L}_{R}$ takes the form

$$
\begin{aligned}
\mathcal{L}_{R} & =\psi_{R}^{\dagger}(x) \Pi_{R}(V) \psi_{R}(x) \\
& =\psi_{R}^{\dagger}(x)\left[i D_{0}-\alpha\left(D_{1}^{2}-D_{2}^{2}\right) \sigma^{1}-\frac{1}{2} \beta\left\{D_{1}, D_{2}\right\} \sigma^{2}+i \gamma D_{3} \sigma^{3}\right] \psi_{R}(x),
\end{aligned}
$$


where $D_{\mu}=\partial_{\mu}+i V_{\mu}(x)$. The function $\mathcal{L}_{R}$ is invariant under local $\mathrm{U}(1)_{R}$ gauge transformations

$$
\text { local } \mathrm{U}(1)_{R}:\left\{\begin{array}{l}
\psi_{R}(x) \longrightarrow e^{i \theta_{R}(x)} \psi_{R}(x) \\
V_{\mu}(x) \longrightarrow V_{\mu}(x)-\partial_{\mu} \theta_{R}(x) .
\end{array}\right.
$$

The operator $\Pi_{R}(V)$ which enters equation (4.1) can be written as the sum of two terms, $\Pi_{R}(V)=\Pi_{R}(0)+\widetilde{\Pi}_{R}(V)$, in which the free part $\Pi_{R}(0)=i \partial_{0}-\alpha\left(\partial_{1}^{2}-\partial_{2}^{2}\right) \sigma^{1}-\beta \partial_{1} \partial_{2} \sigma^{2}+$ $i \gamma \partial_{3} \sigma^{3}$ does not depend on $V_{\mu}$. Therefore the free spinor propagator [1-3] is given by

$$
\begin{aligned}
\widehat{\psi}_{R}(x) \psi_{R}^{\dagger}(y) & \equiv\left\langle 0\left|\mathrm{~T} \psi_{R}(x) \psi_{R}^{\dagger}(y)\right| 0\right\rangle \\
& =i \int \frac{d^{4} p}{(2 \pi)^{4}} e^{-i p(x-y)} \frac{p_{0}+\alpha\left(p_{2}^{2}-p_{1}^{2}\right) \sigma^{1}-\beta p_{1} p_{2} \sigma^{2}-\gamma p_{3} \sigma^{3}}{p_{0}^{2}-\alpha^{2}\left(p_{1}^{2}-p_{2}^{2}\right)^{2}-\beta^{2} p_{1}^{2} p_{2}^{2}-\gamma^{2} p_{3}^{2}+i \varepsilon},
\end{aligned}
$$

and the interaction component of the action takes the form

$$
\begin{aligned}
S_{R I}[V]= & \int d^{4} x \psi_{R}^{\dagger}(x) \widetilde{\Pi}_{R}(V) \psi_{R}(x) \\
= & \int d^{4} x \psi_{R}^{\dagger}(x)\left\{-V_{0}+\alpha\left[V_{1}^{2}-V_{2}^{2}+i\left(\partial_{2} V_{2}\right)+2 i V_{2} \partial_{2}-i\left(\partial_{1} V_{1}\right)-2 i V_{1} \partial_{1}\right] \sigma^{1}\right. \\
& \left.\quad+\frac{1}{2} \beta\left[2 V_{1} V_{2}-i\left(\partial_{1} V_{2}\right)-2 i V_{2} \partial_{1}-i\left(\partial_{2} V_{1}\right)-2 i V_{1} \partial_{2}\right] \sigma^{2}-\gamma V_{3} \sigma^{3}\right\} \psi_{R}(x) .
\end{aligned}
$$

In expression (4.3), the Feynman $\varepsilon$-prescription $[1,3]$ has been introduced in order to guarantee causality and energy positivity. Let $i \Gamma_{R}[V]$ denote the sum of the connected oneloop vacuum-to-vacuum diagrams [1-3] of the $\psi_{R}(x)$ field in the presence of the classical background field $V_{\mu}(x)$,

$$
e^{i \Gamma_{R}[V]}=\left\langle 0\left|\mathrm{~T} e^{i S_{R I}[V]}\right| 0\right\rangle,
$$

where the symbol $\mathrm{T}$ denotes the Wick time-ordering. From the definition (4.5) it follows that, under a gauge transformation $V_{\mu}(x) \rightarrow V_{\mu}(x)-\partial_{\mu} \theta_{R}(x)$, the infinitesimal variation $\delta_{\theta_{R}} \Gamma_{R}[V]$ of $\Gamma_{R}[V]$ is given by the sum of the connected diagrams

$$
\delta_{\theta_{R}} \Gamma_{R}[V]=\int d^{4} x \partial_{\mu} \theta_{R}(x)\left\langle 0\left|\mathrm{~T} J_{R}^{\mu}(x) e^{i S_{R I}}\right| 0\right\rangle^{c}=-\int d^{4} x \theta_{R}(x)\left\langle\partial_{\mu} J_{R}^{\mu}(x)\right\rangle .
$$

The gauge invariance of the lagrangian $\mathcal{L}_{R}$ under the transformations in (4.2) would suggest that $\Gamma_{R}[V]$ also is gauge invariant, and consequently $\delta_{\theta_{R}} \Gamma_{R}[V]=0$. However, because of ultraviolet divergences, the functional $\Gamma_{R}[V]$ is not well defined. Therefore the central question is whether one can define or not a renormalized $\Gamma_{R}[V]$ which is gauge invariant. If a renormalized gauge invariant $\Gamma_{R}[V]$ exists, then the gauge symmetry (4.2) is not anomalous and $\partial_{\mu} J_{R}^{\mu}=0$. Otherwise, $\delta_{\theta_{R}} \Gamma_{R}[V] \neq 0$, and one finds an anomaly

$$
\partial_{\mu} J_{R}^{\mu}(x)=\left\langle\partial_{\mu} J_{R}^{\mu}(x)\right\rangle=\mathcal{P}_{R}(V) \neq 0,
$$

where, in agreement with the action principle, $\mathcal{P}_{R}(V)$ is a local polynomial of the field $V_{\mu}(x)$ and of its space-time derivatives. Usually, the construction of a renormalized $\Gamma_{R}[V]$ consists of two steps: 
1. definition of a regularized functional $\Gamma_{R}^{\mathrm{reg}}[V]$, which depends on a cutoff,

2. introduction of local conterterms $\Gamma_{c t}[V]$, containing in general both divergent and finite parts, which reabsorb the ultraviolet divergences.

The renormalized functional $\Gamma_{R}[V]$, which is well defined (free of divergences), corresponds to the sum $\Gamma_{R}^{\mathrm{reg}}[V]+\Gamma_{c t}[V]$ in the limit in which the cutoff is removed. The particular choice of the regularisation is totally irrelevant. In the renormalization procedure, the freedom of adding finite local counterterms completely removes the dependence of the result on the particular choice of the regularization, because two different regularizations differ (in the limit of removed cutoff) by the sum of finite local counterterms [1-3]. Thus the expression of the polynomial $\mathcal{P}_{R}(V)$ of $V_{\mu}(x)$, which appears in

$$
\delta_{\theta_{R}} \Gamma_{R}[V]=-\int d^{4} x \theta_{R}(x) \mathcal{P}_{R}(V),
$$

is not uniquely determined, because one can add the gauge variation $\delta_{\theta_{R}} L_{c t}[V]$ of some finite local counterterm $L_{c t}[V]$ to the integral $\int \theta_{R} \mathcal{P}_{R}$. Consequently, if $\int \theta_{R} \mathcal{P}_{R}$ can be written as the gauge variation of a local counterterm, then there is no anomaly since, by introducing the appropriate counterterm, one can define a renormalized gauge invariant functional $\Gamma_{R}[V]$. The existence of the anomaly means that expression (4.8) cannot be written as the gauge variation of a local term. In this case, even if one can modify the expression of $\delta_{\theta_{R}} \Gamma_{R}[V]$ - by means of local counterterms - one cannot eliminate $\mathcal{P}_{R}(V)$. Precisely for this reason, the existence of the anomaly does not depend on the choice of the regularization. Of course, the presence or the absence of the anomaly is determined by the lagrangian (4.1) which specifies how the field $\psi_{R}(x)$ interacts with the gauge field.

\subsection{Anomaly as a solution of a cohomological problem}

By developing the consequences of the Wess-Zumino consistency conditions [7], it has been found $[8,64-68]$ that the search of possible nontrivial solutions to equation (4.8) can actually be reduced to a cohomological problem. Indeed, the gauge variation of any function $f\left[V_{\mu}\right]$ can be represented by the action on $f\left[V_{\mu}\right]$ of a nilpotent BRST [69] operator $\mathcal{T}$, which is defined (in the abelian case) by the relations $\mathcal{T} V_{\mu}(x)=-\partial_{\mu} c(x)$ and $\mathcal{T} c(x)=0$, in which the anticommuting variable $c(x)$ takes the place of the gauge parameter $\theta_{R}(x)$. Since the anomaly is determined by the gauge variation of $\Gamma_{R}[V]$, the anomaly is $\mathcal{T}$-closed, but it is not $\mathcal{T}$-exact in the set of local counterterms $\left\{L_{c t}[V]\right\}$. The anomaly then represents a nontrivial solution of the following cohomological problem

$$
\mathcal{T}\left(\int c(x) \mathcal{P}_{R}(V)\right)=0, \quad \int c(x) \mathcal{P}_{R}(V) \neq \mathcal{T} L_{c t}[V], \quad \text { with } \quad \mathcal{T}^{2}=0 .
$$

In this general approach, the gauge fields are described by differential forms, $V=V_{\mu}(x) d x^{\mu}$; no Lorentz invariance is assumed and only the properties of the gauge transformations group enter the solutions. In this way, the possible forms of the gauge anomalies can generally be determined without the need of introducing any corresponding field theory model. More precisely, all the local polynomials of the field $V_{\mu}(x)$, which are not equal 
to the gauge variation of a local counterterm, have been produced. The only parameter which is not fixed à priori by cohomological arguments is the overall normalization factor of each polynomial. The value of this normalization factor is specified by the lagrangian of each particular model.

In the case of the abelian gauge symmetry $V_{\mu}(x) \rightarrow V_{\mu}(x)-\partial_{\mu} \theta_{R}(x)$, equation (4.8) can always [4-6, 70-73] be written in the form

$$
\delta_{\theta_{R}} \Gamma_{R}[V]=-\mathcal{N} \frac{1}{24 \pi^{2}} \int d^{4} x \epsilon^{\mu \nu \tau \lambda} \partial_{\mu} \theta_{R}(x) V_{\nu}(x) \partial_{\tau} V_{\lambda}(x),
$$

where $\mathcal{N}$ represents an overall multiplicative factor that must be computed. If $\mathcal{N}=0$, there is no anomaly. One can easily verify that, when $\mathcal{N} \neq 0$, expression (4.10) cannot be written as the gauge variation of a local counterterm. Therefore the anomaly exists for $\mathcal{N} \neq 0$. In general, the coefficient $\mathcal{N}$ takes integer values; this point will be discussed in section 10. For instance, in the case of a relativistic right-handed Weyl spinor minimally coupled with the gauge field $V_{\mu}(x)$, one finds $\mathcal{N}=1$.

In the case analyzed in this section, the value of $\mathcal{N}$ is determined by the specific form of the lagrangian density (4.1); in particular, the anomaly is specified by the structure of the operator $\Pi_{R}(V)$. By a direct computation, we will show that $\mathcal{N} \neq 0$. Even if in our case the field $\psi_{R}(x)$ does not represent a spinor field, in agreement with the standard notation, expression (4.10) will be called the chiral anomaly.

\subsection{Axial anomaly}

In order to derive the general form of the $\mathrm{U}(1)_{A}$ axial anomaly in the double-Weyl model with lagrangian density (3.5), let us now consider the field $\psi_{L}(x)$ and let us denote by $W_{\mu}(x)$ the gauge field which is coupled with $\psi_{L}(x)$ according to the lagrangian

$$
\begin{aligned}
\mathcal{L}_{L} & =\psi_{L}^{\dagger}(x) \Pi_{L}(W) \psi_{L}(x) \\
& =\psi_{L}^{\dagger}(x)\left[i D_{0}-\alpha\left(D_{1}^{2}-D_{2}^{2}\right) \sigma^{1}-\frac{1}{2} \beta\left\{D_{1}, D_{2}\right\} \sigma^{2}-i \gamma D_{3} \sigma^{3}\right] \psi_{L}(x),
\end{aligned}
$$

where $D_{\mu}=\partial_{\mu}+i W_{\mu}(x) . \mathcal{L}_{L}$ is invariant under local $\mathrm{U}(1)_{L}$ gauge transformations

$$
\text { local } \mathrm{U}(1)_{L}:\left\{\begin{array}{l}
\psi_{L}(x) \longrightarrow e^{i \theta_{L}(x)} \psi_{L}(x) \\
W_{\mu}(x) \longrightarrow W_{\mu}(x)-\partial_{\mu} \theta_{L}(x) .
\end{array}\right.
$$

Let $i \Gamma_{L}[W]$ be the sum of the connected one-loop vacuum-to-vacuum diagrams of $\psi_{L}(x)$ in the presence of the classical field $W_{\mu}$. The infinitesimal variation $\delta_{\theta_{L}} \Gamma_{L}[W]$ of $\Gamma_{L}[W]$ under the transformation $W_{\mu}(x) \rightarrow W_{\mu}(x)-\partial_{\mu} \theta_{L}(x)$ is strictly related with $\delta_{\theta_{R}} \Gamma_{R}[V]$. Indeed, the lagrangian for $\psi_{L}(x)$ can be obtained from the lagrangian for $\psi_{R}(x)$ by means of the substitution $\sigma_{3} \rightarrow-\sigma_{3}$. This means that the expression of the anomaly for $\psi_{L}(x)$ can be obtained from expression (4.10) provided we introduce, in addition to the obvious change of variables, a change of the sign of the $x^{3}$-derivative, $\partial_{3} \rightarrow-\partial_{3}$, and a change of the sign 
of the third component of the field $W_{\mu}(x), W_{3}(x) \rightarrow-W_{3}(x)$. Therefore

$$
\delta_{\theta_{L}} \Gamma_{L}[W]=\mathcal{N} \frac{1}{24 \pi^{2}} \int d^{4} x \epsilon^{\mu \nu \tau \lambda} \partial_{\mu} \theta_{L}(x) W_{\nu}(x) \partial_{\tau} W_{\lambda}(x) .
$$

Let us now consider the complete model with lagrangian (3.5) in which both $\psi_{R}(x)$ and $\psi_{L}(x)$ are present. The gauge fields $V_{\mu}(x)$ and $W_{\mu}(x)$, that refer to the components of the group $\mathrm{U}(1)_{R} \times \mathrm{U}(1)_{L}$, can be written as combinations of the vector fields associated with the components of $\mathrm{U}(1)_{V} \times \mathrm{U}(1)_{A}$ :

$$
\begin{aligned}
\mathrm{U}(1)_{V}, & A_{\mu}(x) & =\frac{1}{2}\left[V_{\mu}(x)+W_{\mu}(x)\right] \\
\mathrm{U}(1)_{A}, & B_{\mu}(x) & =\frac{1}{2}\left[V_{\mu}(x)-W_{\mu}(x)\right] .
\end{aligned}
$$

The infinitesimal variation of $\widetilde{\Gamma}[A, B]=\Gamma_{R}[V(A, B)]+\Gamma_{L}[W(A, B)]$ under the vector $\mathrm{U}(1)_{V}$ transformation $A_{\mu}(x) \rightarrow A_{\mu}(x)-\partial_{\mu} \theta_{V}(x)$ is obtained by combining equations (4.10) and (4.13)

$$
\delta_{\theta_{V}} \widetilde{\Gamma}[A, B]=-\mathcal{N} \frac{1}{6 \pi^{2}} \int d^{4} x \epsilon^{\mu \nu \tau \lambda} \partial_{\mu} \theta_{V}(x) B_{\nu}(x) \partial_{\tau} A_{\lambda}(x),
$$

while the infinitesimal variation of $\widetilde{\Gamma}[A, B]$ under the $\mathrm{U}(1)_{A}$ axial transformation $B_{\mu}(x) \rightarrow$ $B_{\mu}(x)-\partial_{\mu} \theta_{A}(x)$ turns out to be

$$
\delta_{\theta_{A}} \widetilde{\Gamma}[A, B]=-\mathcal{N} \frac{1}{12 \pi^{2}} \int d^{4} x \epsilon^{\mu \nu \tau \lambda} \partial_{\mu} \theta_{A}(x)\left[A_{\nu}(x) \partial_{\tau} A_{\lambda}(x)+B_{\nu}(x) \partial_{\tau} B_{\lambda}(x)\right] .
$$

Let us introduce the functional

$$
\Gamma[A, B]=\widetilde{\Gamma}[A, B]+L[A, B]
$$

where the finite local counterterm $L[A, B]$ is given by

$$
L[A, B]=-\mathcal{N} \frac{1}{6 \pi^{2}} \int d^{4} x \epsilon^{\mu \nu \tau \lambda} A_{\mu}(x) B_{\nu}(x) \partial_{\tau} A_{\lambda}(x) .
$$

The infinitesimal variations of $\Gamma[A, B]$ under $\mathrm{U}(1)_{V} \times \mathrm{U}(1)_{A}$ transformations take the form

$$
\delta_{\theta_{V}} \Gamma[A, B]=0,
$$

and

$$
\delta_{\theta_{A}} \Gamma[A, B]=\mathcal{N} \frac{1}{12 \pi^{2}} \int d^{4} x \epsilon^{\mu \nu \tau \lambda} \theta_{A}(x)\left[\partial_{\mu} B_{\nu}(x) \partial_{\tau} B_{\lambda}(x)+3 \partial_{\mu} A_{\nu}(x) \partial_{\tau} A_{\lambda}(x)\right] .
$$

One can easily verify that expression (4.20) is not the gauge variation of a local counterterm. Equation (4.19) shows that the subgroup $\mathrm{U}(1)_{V}$ is anomaly free; consequently, the vector gauge invariance (3.2) is preserved and the corresponding local gauge theory is consistent. The gauge anomaly only concerns the axial subgroup $\mathrm{U}(1)_{A}$. In the model which is described by the lagrangian density (3.5), the field $B_{\mu}(x)$ is vanishing; therefore expression (4.20) evaluated at $B_{\mu}(x)=0$ gives

$$
\left.\delta_{\theta_{A}} \Gamma[A, B]\right|_{B_{\mu}=0}=\mathcal{N} \frac{1}{4 \pi^{2}} \int d^{4} x \epsilon^{\mu \nu \tau \lambda} \theta_{A}(x) \partial_{\mu} A_{\nu}(x) \partial_{\tau} A_{\lambda}(x) .
$$


So, in the double-Weyl model (3.5), the divergence of the axial current — or the expression of the axial anomaly - takes the form

$$
\partial_{\mu} J_{A}^{\mu}(x)=-\mathcal{N} \frac{1}{4 \pi^{2}} \epsilon^{\mu \nu \tau \lambda} \partial_{\mu} A_{\nu}(x) \partial_{\tau} A_{\lambda}(x),
$$

which is in agreement with equation (3.9); the value of $\mathcal{N}$ remains to be computed.

Equation (4.22) shows that, in multi-Weyl semimetals as well as in other generic field theory models, the axial anomaly — if present — is proportional to the standard axial anomaly of massless electrodynamics. Indeed, on the one hand, the cohomological problem (4.9) admits a universal nontrivial solution and, on the other hand, in the presence of $\mathrm{U}(1)_{R} \times \mathrm{U}(1)_{R}$ symmetry the vector $\mathrm{U}(1)_{V}$ invariance is required to be preserved. Thus no functional modification - due to the absence of effective Lorentz covariance of the classical lagrangian - appears in the axial anomaly.

We mention finally that if an explicit gauge symmetry breaking is induced, modifications from the expression in (4.22) are expected; one example has been considered recently in [74].

\section{Perturbative computation}

In this section we shall derive the expression (4.10) of the chiral anomaly for the doubleWeyl model by means of perturbation theory. In particular, the value of $\mathcal{N}$ - appearing in equation (4.10) - will be determined. This means that, as it has been shown in section 4 , the result of this section provides a proof of equation (3.9).

\subsection{Regularization}

As it has been shown in section 4 , the origin of the chiral anomaly is represented by the nontriviality of the gauge variation (4.10) of the functional $\Gamma_{R}[V]$. The sum of the connected one-loop diagrams entering the definition (4.5) is given by [1-3]

$$
\begin{aligned}
i \Gamma_{R}[V] & =-\sum_{n=1}^{\infty} \frac{1}{n} \operatorname{Tr}\left[i \psi_{R} \psi_{R}^{\dagger} \widetilde{\Pi}_{R}(V)\right]^{n} \\
& =-\sum_{n=1}^{\infty} \frac{1}{n} \operatorname{Tr}\left[\int d^{4} x_{1} \ldots d^{4} x_{n}\left\langle x_{1}\left|i \bar{\psi}_{R} \psi_{R}^{\dagger} \widetilde{\Pi}_{R}(V)\right| x_{2}\right\rangle \cdots\left\langle x_{n}\left|i{\overrightarrow{\psi_{R}}}_{\psi_{R}}^{\dagger} \widetilde{\Pi}_{R}(V)\right| x_{1}\right\rangle\right]
\end{aligned}
$$

where, in agreement with the Schwinger notations [4], the symbol Tr represents the trace

$$
\operatorname{Tr}(Q)=\int d^{4} x \operatorname{tr}\langle x|Q| x\rangle,
$$

in which Tr denotes the trace over the indices of the sigma matrices. Since the fermion propagator takes the form shown in equation (4.3), equality (5.2) can be written as

$$
i \Gamma_{R}[V]=\operatorname{Tr} \ln \left\{1+\frac{i \partial_{0}+\alpha\left(\partial_{1}^{2}-\partial_{2}^{2}\right) \sigma^{1}+\beta \partial_{1} \partial_{2} \sigma^{2}-i \gamma \partial_{3} \sigma^{3}}{-\partial_{0}^{2}-\alpha^{2}\left(\partial_{1}^{2}-\partial_{2}^{2}\right)^{2}-\beta^{2} \partial_{1}^{2} \partial_{2}^{2}+\gamma^{2} \partial_{3}^{2}+i \varepsilon} \widetilde{\Pi}_{R}(V)\right\}
$$


Indeed the expansion of expression (5.3) in powers of $\widetilde{\Pi}_{R}(V)$ coincides with equation (5.2). The terms of the sum (5.2) which correspond to the divergent Feynman diagrams are not well defined. So we now introduce a regularisation. Let us recall that, if $y$ is a positive number, one has

$$
\ln y+\text { constant }=-\lim _{\epsilon \rightarrow 0} \int_{\epsilon}^{\infty} \frac{d s}{s} e^{-s y} .
$$

Therefore, according to the Schwinger proper-time regularisation [4], the regularised oneloop functional is defined as

$$
\Gamma_{R}^{\mathrm{reg}}[V]=i \int_{\epsilon}^{\infty} \frac{d s}{s} \operatorname{Tr}\left[e^{s \Sigma \Pi_{R}(V)}\right]+\text { constant },
$$

where the constant does not depend on $V_{\mu}, \Pi_{R}(V)$ is shown in equation (4.1), and the $\Sigma$ operator,

$$
\Sigma=i \partial_{0}+\alpha\left(\partial_{1}^{2}-\partial_{2}^{2}\right) \sigma^{1}+\beta \partial_{1} \partial_{2} \sigma^{2}-i \gamma \partial_{3} \sigma^{3},
$$

enters the definition of the propagator (4.3). The sign in the exponent of equation (5.5) is fixed by the positivity of the analytic extension of $-\Sigma \Pi_{R}(V)$ in the euclidean region for the momenta. The parameter $\epsilon>0$ represents the cut-off, and the limit of vanishing cut-off is obtained by taking the $\epsilon \rightarrow 0$ limit.

\subsection{Gauge variation}

Under a gauge transformation $V_{\mu}(x) \rightarrow V_{\mu}(x)-\partial_{\mu} \theta_{R}(x)$, the infinitesimal variation of $\Gamma_{R}^{\mathrm{reg}}[V]$ is given by

$$
\begin{aligned}
& \delta_{\theta_{R}} \Gamma_{R}^{\mathrm{reg}}[V]=i \int_{\epsilon}^{\infty} \frac{d s}{s}(i s) \operatorname{Tr}\left\{e^{s \Sigma \Pi_{R}[V]} \Sigma\left[\theta_{R}, \Pi_{R}(V)\right]\right\} \\
& =\int_{\epsilon}^{\infty} d s \operatorname{Tr}\left\{e^{s \Sigma \Pi_{R}} \Sigma \Pi_{R} \theta_{R}-e^{s \Pi_{R} \Sigma} \Pi_{R} \Sigma \theta_{R}\right\}=\operatorname{Tr}\left\{\left[e^{\epsilon \Sigma \Pi_{R}[V]}-e^{\epsilon \Pi_{R}[V] \Sigma}\right] \theta_{R}\right\} .
\end{aligned}
$$

By means of the relation

$$
\begin{aligned}
e^{X+Y}= & e^{X}+\int_{0}^{1} d u e^{(1-u) X} Y e^{u X}+\int_{0}^{1} u d u \int_{0}^{1} d v e^{(1-u) X} Y e^{u(1-v) X} Y e^{u v X} \\
& +\int_{0}^{1} u^{2} d u \int_{0}^{1} v d v \int_{0}^{1} d t e^{(1-u) X} Y e^{u(1-v) X} Y e^{u v(1-t) X} Y e^{u v t X}+\cdots
\end{aligned}
$$

one obtains

$$
\begin{aligned}
\delta_{\theta_{R}} \Gamma_{R}^{\mathrm{reg}}[V]=\operatorname{Tr}\{ & \epsilon \int_{0}^{1} d u e^{(1-u) \epsilon \Pi^{2}} \widetilde{\Pi}_{R}[V] e^{u \epsilon \Pi^{2}}\left[\theta_{R}, \Sigma\right] \\
& +\epsilon^{2} \int_{0}^{1} u d u \int_{0}^{1} d v e^{(1-u) \epsilon \Pi^{2}} \widetilde{\Pi}_{R}[V] e^{u(1-v) \epsilon \Pi^{2}} \Sigma \widetilde{\Pi}_{R}[V] e^{u v \epsilon \Pi^{2}}\left[\theta_{R}, \Sigma\right] \\
& +\epsilon^{3} \int_{0}^{1} u^{2} d u \int_{0}^{1} v d v \int_{0}^{1} d t e^{(1-u) \epsilon \Pi^{2}} \widetilde{\Pi}_{R}[V] e^{u(1-v) \epsilon \Pi^{2}} \Sigma \widetilde{\Pi}_{R}[V] \times \\
& \left.\times e^{u v(1-t) \epsilon \Pi^{2}} \Sigma \widetilde{\Pi}_{R}[V] e^{u v t \epsilon \Pi^{2}}\left[\theta_{R}, \Sigma\right]+\cdots\right\}
\end{aligned}
$$


where

$$
\Pi^{2}=-\partial_{0}^{2}-\alpha^{2}\left(\partial_{1}^{2}-\partial_{2}^{2}\right)^{2}-\beta^{2} \partial_{1}^{2} \partial_{2}^{2}+\gamma^{2} \partial_{3}^{2},
$$

and $\widetilde{\Pi}_{R}(V)$ is shown in equation (4.4). Note that $\Pi^{2}$ is symmetric under the exchange $\partial_{1} \leftrightarrow \partial_{2}$.

\subsection{Computation rules}

The trace (5.9) is computed by moving all the space-time derivatives on the right and the terms which do not contain derivatives on the left so that

$$
\operatorname{Tr}\{F(x) G(i \partial)\}=\int d^{4} x \int \frac{d^{4} p}{(2 \pi)^{4}} \operatorname{Tr}\{F(x) G(p)\},
$$

where the correspondence $i \partial_{\mu} \rightarrow p_{\mu}$ has been used. Since $\Pi^{2}$ contains $p_{0}$ and $p_{3}$ at power 2 , and $p_{1}$ and $p_{2}$ at power 4 , the integration over the momenta in the euclidean region gives rise to the following powers of $\epsilon$

$$
\int d^{4} p e^{\epsilon \Pi^{2}}\left[p_{0,3}\right]^{a}\left[p_{1,2}\right]^{b} \sim \epsilon^{-3 / 2-a / 2-b / 4} .
$$

In the $\epsilon \rightarrow 0$ limit, expression (5.9) is a sum of a large number of nonvanishing contributions. Many of these contributions do not play a part in the anomaly because they are just equal to the variation of local counterterms. So, let us concentrate on the relevant (as far as the anomaly is concerned) terms which are of the type

$$
\text { relevant terms } \sim \partial_{\mu} \theta_{R}(x) V_{\nu}(x) \partial_{\tau} V_{\lambda}(x), \quad(\text { with } \quad \mu \neq \nu \neq \tau \neq \lambda)
$$

in which there is not a couple of the indices $\mu, \nu, \tau, \lambda$ which assume the same value. Let $\Gamma_{c t}[V]$ be the sum of the local counterterms whose gauge variation cancels precisely the integrable contributions of $\delta_{\theta_{R}} \Gamma_{R}^{\mathrm{reg}}[V]$ which are not of the type (5.13). With the definition $\Gamma_{R}[V]=\Gamma_{R}^{\mathrm{reg}}[V]-\Gamma_{c t}[V]$, we shall now consider the gauge variation of $\Gamma_{R}[V]$ in the $\epsilon \rightarrow 0$ limit.

There are $4 !=24$ contributions of type (5.13), which are contained in the $\epsilon^{2}$ term of the expansion (5.9). In order to obtain a nonvanishing result in the $\epsilon \rightarrow 0$ limit, one needs to compensate the powers of the cut-off $\epsilon$ by powers of the momenta in the integrals (5.11) and (5.12). We will need to extract powers of the momenta also from the exponential factors of the type $e^{q \epsilon \Pi^{2}}$. More precisely, when one exponential factor $e^{q \epsilon \Pi^{2}}$ commutes with a function $f(x)$, it gives the expression

$$
\begin{aligned}
{\left[e^{q \epsilon \Pi^{2}}, f(x)\right]=-\epsilon q } & \left\{2 \partial_{0} f(x) \partial_{0}-2 \gamma^{2} \partial_{3} f(x) \partial_{3}+4 \alpha^{2}\left(\partial_{1} f(x) \partial_{1}^{3}+\partial_{2} f(x) \partial_{2}^{3}\right)\right. \\
+ & \left.2\left(\beta^{2}-2 \alpha^{2}\right)\left(\partial_{1} f(x) \partial_{1} \partial_{2}^{2}+\partial_{2} f(x) \partial_{1}^{2} \partial_{2}\right)\right\} e^{q \epsilon \Pi^{2}}+\cdots
\end{aligned}
$$

where, in agreement with relation (5.12), the first two terms give rise to contributions of order $\epsilon^{1 / 2}$,

$$
-\epsilon q\left\{2 \partial_{0} f(x) \partial_{0}-2 \gamma^{2} \partial_{3} f(x) \partial_{3}\right\} \rightarrow \epsilon^{1 / 2}
$$




\begin{tabular}{|c|c|c|c|c|c|}
\hline$c_{n}$ & $F_{n}(x)$ & $G_{n}(p)$ & $c_{n}$ & $F_{n}(x)$ & $G_{n}(p)$ \\
\hline 1 & $V_{0} \partial_{1} \theta_{R} \partial_{2} V_{3}$ & $\alpha^{2} p_{1}^{6}+\left(\beta^{2}-\alpha^{2}\right) p_{1}^{4} p_{2}^{2}$ & 1 & $V_{2} \partial_{0} \theta_{R} \partial_{1} V_{3}$ & $\alpha^{2} p_{1}^{6}+\left(\beta^{2}-\alpha^{2}\right) p_{1}^{4} p^{2}$ \\
\hline$-2 \gamma^{2}$ & $V_{0} \partial_{1} \theta_{R} \partial_{3} V_{2}$ & $p_{1}^{2} p_{3}^{2}$ & $-2 \gamma^{2}$ & $V_{2} \partial_{0} \theta_{R} \partial_{3} V_{1}$ & $p_{1}^{2} p_{3}^{2}$ \\
\hline $2 \gamma^{2}$ & $V_{0} \partial_{2} \theta_{R} \partial_{3} V_{1}$ & $p_{1}^{2} p_{3}^{2}$ & $2 \gamma^{2}$ & $V_{2} \partial_{1} \theta_{R} \partial_{3} V_{0}$ & $p_{1}^{2} p_{3}^{2}$ \\
\hline-1 & $V_{0} \partial_{2} \theta_{R} \partial_{1} V_{3}$ & $\alpha^{2} p_{1}^{6}+\left(\beta^{2}-\alpha^{2}\right) p_{1}^{4} p_{2}^{2}$ & 2 & $V_{2} \partial_{1} \theta_{R} \partial_{0} V_{3}$ & $p_{1}^{2} p_{0}^{2}$ \\
\hline 1 & $V_{0} \partial_{3} \theta_{R} \partial_{1} V_{2}$ & $\alpha^{2} p_{1}^{6}+\left(\beta^{2}-\alpha^{2}\right) p_{1}^{4} p_{2}^{2}$ & -2 & $V_{2} \partial_{3} \theta_{R} \partial_{0} V_{1}$ & $p_{1}^{2} p_{0}^{2}$ \\
\hline-1 & $V_{0} \partial_{3} \theta_{R} \partial_{2} V_{1}$ & $\alpha^{2} p_{1}^{6}+\left(\beta^{2}-\alpha^{2}\right) p_{1}^{4} p_{2}^{2}$ & -1 & $V_{2} \partial_{3} \theta_{R} \partial_{1} V_{0}$ & $\alpha^{2} p_{1}^{6}+\left(\beta^{2}-\alpha^{2}\right) p_{1}^{4} p_{2}^{2}$ \\
\hline-2 & $V_{1} \partial_{2} \theta_{R} \partial_{0} V_{3}$ & $p_{1}^{2} p_{0}^{2}$ & 1 & $V_{3} \partial_{2} \theta_{R} \partial_{1} V_{0}$ & $\alpha^{2} p_{1}^{6}+\left(\beta^{2}-\alpha^{2}\right) p_{1}^{4} p_{2}^{2}$ \\
\hline$-2 \gamma^{2}$ & $V_{1} \partial_{2} \theta_{R} \partial_{3} V_{0}$ & $p_{1}^{2} p_{3}^{2}$ & 2 & $V_{3} \partial_{2} \theta_{R} \partial_{0} V_{1}$ & $p_{1}^{2} p_{0}^{2}$ \\
\hline $2 \gamma^{2}$ & $V_{1} \partial_{0} \theta_{R} \partial_{3} V_{2}$ & $p_{1}^{2} p_{3}^{2}$ & -2 & $V_{3} \partial_{1} \theta_{R} \partial_{0} V_{2}$ & $p_{1}^{2} p_{0}^{2}$ \\
\hline-1 & $V_{1} \partial_{0} \theta_{R} \partial_{2} V_{3}$ & $\alpha^{2} p_{1}^{6}+\left(\beta^{2}-\alpha^{2}\right) p_{1}^{4} p_{2}^{2}$ & -1 & $V_{3} \partial_{1} \theta_{R} \partial_{2} V_{0}$ & $\alpha^{2} p_{1}^{6}+\left(\beta^{2}-\alpha^{2}\right) p_{1}^{4} p_{2}^{2}$ \\
\hline 1 & $V_{1} \partial_{3} \theta_{R} \partial_{2} V_{0}$ & $\alpha^{2} p_{1}^{6}+\left(\beta^{2}-\alpha^{2}\right) p_{1}^{4} p_{2}^{2}$ & 1 & $V_{3} \partial_{0} \theta_{R} \partial_{2} V_{1}$ & $\alpha^{2} p_{1}^{6}+\left(\beta^{2}-\alpha^{2}\right) p_{1}^{4} p_{2}^{2}$ \\
\hline 2 & $V_{1} \partial_{3} \theta_{R} \partial_{0} V_{2}$ & $p_{1}^{2} p_{0}^{2}$ & -1 & $V_{3} \partial_{0} \theta_{R} \partial_{1} V_{2}$ & $\alpha^{2} p_{1}^{6}+\left(\beta^{2}-\alpha^{2}\right) p_{1}^{4} p_{2}^{2}$ \\
\hline
\end{tabular}

Table 1. Addenda in the sum of eq. (5.17).

whereas the remaining two terms give rise to contributions of order $\epsilon^{1 / 4}$

$$
-\epsilon q\left\{4 \alpha^{2}\left(\partial_{1} f(x) \partial_{1}^{3}+\partial_{2} f(x) \partial_{2}^{3}\right)+2\left(\beta^{2}-2 \alpha^{2}\right)\left(\partial_{1} f(x) \partial_{1} \partial_{2}^{2}+\partial_{2} f(x) \partial_{1}^{2} \partial_{2}\right)\right\} \longrightarrow \epsilon^{1 / 4},
$$

and the dots stand for terms which turn out to be irrelevant (they produce vanishing outcomes in the $\epsilon \rightarrow 0$ limit).

\subsection{Addition of the contributions}

We have found 144 nonvanishing contributions to $\delta_{\theta_{R}} \Gamma_{R}[V]$ and their sum can be written in the form

$$
\delta_{\theta_{R}} \Gamma_{R}[V]=\frac{-i 8 \alpha \beta \gamma \epsilon^{3}}{3} \sum_{n} c_{n} \int d^{4} x F_{n}(x) \int \frac{d^{4} p}{(2 \pi)^{4}} e^{\epsilon \Pi^{2}} G_{n}(p)
$$

where the sum contains 24 addenda and the values of $c_{n}, F_{n}(x)$ and $G_{n}(p)$ are shown in table 1.

Let us recall that in momentum space $\left(i \partial_{\mu} \rightarrow p_{\mu}\right)$ one has

$$
e^{\epsilon \Pi^{2}}=e^{\epsilon p_{0}^{2}} e^{-\epsilon \gamma^{2} p_{3}^{2}} e^{-\epsilon\left[\alpha^{2}\left(p_{1}^{2}-p_{2}^{2}\right)^{2}+\beta^{2} p_{1}^{2} p_{2}^{2}\right]} .
$$

In agreement with the Feynman $\varepsilon$-convention of the propagator, the analytic continuation in the euclidean region is obtained according to $p_{0} \rightarrow i p_{4}$ with real $p_{4}$. One gets

$$
\int d p_{4} e^{-\epsilon p_{4}^{2}}=\pi^{1 / 2} \epsilon^{-1 / 2}, \quad \int d p_{4} e^{-\epsilon p_{4}^{2}} p_{4}^{2}=\frac{1}{2} \pi^{1 / 2} \epsilon^{-3 / 2},
$$

and

$$
\int d p_{3} e^{-\epsilon \gamma^{2} p_{3}^{2}}=\pi^{1 / 2} \epsilon^{-1 / 2} \frac{1}{|\gamma|}, \quad \int d p_{3} e^{-\epsilon \gamma^{2} p_{3}^{2}} p_{3}^{2}=\frac{1}{2} \pi^{1 / 2} \epsilon^{-3 / 2} \frac{1}{|\gamma|^{3}} .
$$


Let us define

$$
\int d p_{1} d p_{2} e^{-\epsilon\left[\alpha^{2}\left(p_{1}^{2}-p_{2}^{2}\right)^{2}+\beta^{2} p_{1}^{2} p_{2}^{2}\right]} p_{1}^{2}=\pi \epsilon^{-1} X(\alpha, \beta)
$$

Since

$$
\begin{aligned}
&-\frac{\partial}{\partial \epsilon} \int d p_{1} d p_{2} e^{-\epsilon\left[\alpha^{2}\left(p_{1}^{2}-p_{2}^{2}\right)^{2}+\beta^{2} p_{1}^{2} p_{2}^{2}\right]} p_{1}^{2}= \\
&=\int d p_{1} d p_{2} e^{-\epsilon\left[\alpha^{2}\left(p_{1}^{2}-p_{2}^{2}\right)^{2}+\beta^{2} p_{1}^{2} p_{2}^{2}\right]}\left[\alpha^{2} p_{1}^{6}+\left(\beta^{2}-\alpha^{2}\right) p_{1}^{4} p_{2}^{2}\right]
\end{aligned}
$$

from equations (5.21) and (5.22) one derives

$$
\int d p_{1} d p_{2} e^{-\epsilon\left[\alpha^{2}\left(p_{1}^{2}-p_{2}^{2}\right)^{2}+\beta^{2} p_{1}^{2} p_{2}^{2}\right]}\left[\alpha^{2} p_{1}^{6}+\left(\beta^{2}-\alpha^{2}\right) p_{1}^{4} p_{2}^{2}\right]=\pi \epsilon^{-2} X(\alpha, \beta) .
$$

Therefore, the momenta integrals with appear in equation (5.17) take the values

$$
\begin{array}{r}
\int \frac{d^{4} p}{\left(2 \pi^{4}\right)} e^{\epsilon \Pi^{2}} p_{1}^{2} p_{3}^{2} \longrightarrow \epsilon^{-3}\left(\frac{i}{32 \pi^{2}}\right) \frac{1}{|\gamma|^{3}} X(\alpha, \beta), \\
\int \frac{d^{4} p}{\left(2 \pi^{4}\right)} e^{\epsilon \Pi^{2}} p_{1}^{2} p_{0}^{2} \longrightarrow \epsilon^{-3}\left(\frac{-i}{32 \pi^{2}}\right) \frac{1}{|\gamma|} X(\alpha, \beta), \\
\int \frac{d^{4} p}{\left(2 \pi^{4}\right)} e^{\epsilon \Pi^{2}}\left(\alpha^{2} p_{1}^{6}+\left(\beta^{2}-\alpha^{2}\right) p_{1}^{4} p_{2}^{2}\right) \longrightarrow \epsilon^{-3}\left(\frac{i}{16 \pi^{2}}\right) \frac{1}{|\gamma|} X(\alpha, \beta) .
\end{array}
$$

Consequently, the sum (5.17) is given by

$$
\delta_{\theta_{R}} \Gamma_{R}[V]=-\frac{\alpha \beta \gamma X(\alpha, \beta)}{6 \pi^{2}|\gamma|} \int d^{4} x \epsilon^{\mu \nu \tau \lambda} \partial_{\mu} \theta_{R}(x) V_{\nu}(x) \partial_{\tau} V_{\lambda}(x) .
$$

Even if the fermion operator $\Pi_{R}(V)$ is not Lorentz covariant and differs from the standard Dirac operator, and even if $\Pi_{R}(V)$ contains dimensioned parameters, still the sum of the various contributions to $\delta_{\theta_{R}} \Gamma_{R}[V]$ - quite remarkably - reproduces the standard form

$$
F \wedge F=\left(\partial_{\mu} V_{\nu}-\partial_{\nu} V_{\mu}\right)\left(\partial_{\lambda} V_{\tau}-\partial_{\tau} V_{\lambda}\right) d x^{\mu} \wedge d x^{\nu} \wedge d x^{\lambda} \wedge d x^{\tau}
$$

of the chiral anomaly (4.10). As it has been mentioned in section 4, this is a consequence of the fact that, in the abelian case, the nontrivial solution of the cohomological problem (4.9) is given precisely by $F \wedge F$.

\subsection{The final result}

Let us now derive the value of $X(\alpha, \beta)$. By means of a rescaling of the integration variables $p_{i} \rightarrow p_{i} / \epsilon^{1 / 4}$, equation (5.21) can be written as

$$
X(\alpha, \beta)=\frac{1}{2 \pi} \int d p_{1} d p_{2} e^{-\left[\alpha^{2}\left(p_{1}^{2}+p_{2}^{2}\right)^{2}+\left(\beta^{2}-4 \alpha^{2}\right) p_{1}^{2} p_{2}^{2}\right]}\left(p_{1}^{2}+p_{2}^{2}\right) .
$$

By introducing two dimensional spherical coordinates, $p_{1}=p \cos \varphi, p_{2}=p \sin \varphi$, one finds

$$
\alpha^{2}\left(p_{1}^{2}+p_{2}^{2}\right)^{2}+\left(\beta^{2}-4 \alpha^{2}\right) p_{1}^{2} p_{2}^{2}=\frac{p^{4}}{8}\left[\left(4 \alpha^{2}+\beta^{2}\right)+\left(4 \alpha^{2}-\beta^{2}\right) \cos (4 \varphi)\right]
$$


and then

$$
\begin{aligned}
X(\alpha, \beta) & =\frac{1}{8 \pi} \int_{0}^{2 \pi} d \varphi \frac{8}{4 \alpha^{2}+4 \beta^{2}+\left(4 \alpha^{2}-\beta^{2}\right) \cos (4 \varphi)} \\
& =\frac{4}{\pi \sqrt{\left(4 \alpha^{2}+\beta^{2}\right)^{2}-\left(4 \alpha^{2}-\beta^{2}\right)^{2}}} \arctan \left[\frac{\left(1-\frac{4 \alpha^{2}-\beta^{2}}{4 \alpha^{2}+\beta^{2}}\right) \tan (\theta / 2)}{\sqrt{1-\left(\frac{4 \alpha^{2}-\beta^{2}}{4 \alpha^{2}+\beta^{2}}\right)^{2}}}\right]_{0}^{\pi} \\
& =\frac{1}{2|\alpha \beta|}
\end{aligned}
$$

Equation (5.27) then reads

$$
\delta_{\theta_{R}} \Gamma_{R}[V]=-\frac{2 \alpha \beta \gamma}{|\alpha \beta \gamma|} \frac{1}{24 \pi^{2}} \int d^{4} x \epsilon^{\mu \nu \tau \lambda} \partial_{\mu} \theta_{R}(x) V_{\nu}(x) \partial_{\tau} V_{\lambda}(x) .
$$

This means that, in equation (4.10), the multiplicative factor $\mathcal{N}$ is given by

$$
\mathcal{N}=2 \frac{\alpha \beta \gamma}{|\alpha \beta \gamma|}
$$

Therefore, in the double-Weyl model specified by the lagrangian (3.5), the value of the axial anomaly (4.22) coincides with expression (3.9). This concludes the perturbative quantum field theory proof of equations (3.9) and (3.10).

\section{Nielsen-Ninomiya procedure}

When the vector gauge invariance (3.2) is preserved, in the presence of appropriate electric and magnetic fields, the axial anomaly can be interpreted as the rate of production of "particles chirality" as a consequence of a vacuum rearrangement [14] for the fermions. Nielsen and Ninomiya showed that the axial anomaly can be estimated by considering a simple quantum mechanical description of the system. In the presence of a uniform magnetic field along the $x^{3}$ direction, the spectrum of the hamiltonian associated with a Weyl cone displays a three-dimensional Landau level structure. Among the gapped Landau level, there is a special gapless family of states with a chiral dispersion along the $x^{3}$ direction. This family of states is responsible for chiral anomaly upon the application of an electric field along $x^{3}$. Based on this idea, in the present section we shall rederive the result (3.9). The main step will be to define the gapless and chiral families of states appearing when the Weyl cones are subject to a suitable magnetic field.

\subsection{Classical external fields}

Let us consider the case in which classical electric and magnetic fields $E$ and $B$ are directed in the $x^{3}$ direction,

$$
A_{0}(x)=0, \quad A_{1}(x)=0, \quad A_{2}(x)=-B x^{1}, \quad A_{3}(x)=E x^{0},
$$


with $E>0$ and $B>0$. The equation of motion for the field $\psi_{R}(x)$ takes the form

$$
\left\{i \partial_{0}-\alpha\left[\partial_{1}^{2}-\left(\partial_{2}-i B x^{1}\right)^{2}\right] \sigma^{1}-\frac{\beta}{2}\left\{\partial_{1},\left(\partial_{2}-i B x^{1}\right)\right\} \sigma^{2}+i \gamma\left(\partial_{3}+i E x^{0}\right) \sigma^{3}\right\} \psi_{R}(x)=0 .
$$

This equation of motion is translationally invariant along $x^{2}$ and $x^{3}$, so that we can consider, in full generality, wavefunctions with specific eigenvalues $k_{2}$ and $k_{3}$ of the two components $p_{2}$ and $p_{3}$ of the momentum:

$$
\psi_{R}(x)=e^{i k_{2} x^{2}} e^{i k_{3} x^{3}} \chi\left(x^{0}, x^{1}\right)=e^{i k_{2} x^{2}} e^{i k_{3} x^{3}}\left(\begin{array}{c}
\chi_{\uparrow}\left(x^{0}, x^{1}\right) \\
\chi_{\downarrow}\left(x^{0}, x^{1}\right)
\end{array}\right) .
$$

It is useful to introduce the operators

$$
\eta=\frac{1}{\sqrt{2 B}} \partial_{1}+\sqrt{\frac{B}{2}}\left(x^{1}-\frac{k_{2}}{B}\right), \quad \eta^{\dagger}=-\frac{1}{\sqrt{2 B}} \partial_{1}+\sqrt{\frac{B}{2}}\left(x^{1}-\frac{k_{2}}{B}\right),
$$

which satisfy the standard commutation relation of annihilation and creation operators, $\left[\eta, \eta^{\dagger}\right]=1$. The associated wavefunction

$$
h_{0}\left(x^{1}\right)=(B / \pi)^{1 / 4} \exp \left[-\frac{B}{2}\left(x^{1}-k_{2} / B\right)^{2}\right]
$$

corresponds to the ground state of the 1D harmonic oscillator in the $x^{1}$ direction centered in $k_{2} / B$ [75] such that $\eta h_{0}=0$. Equation (6.2) allows us to define the hamiltonian of the system for the right modes:

$$
\begin{aligned}
i \partial_{0}\left(\begin{array}{c}
\chi_{\uparrow} \\
\chi_{\downarrow}
\end{array}\right) & =\left(\begin{array}{cc}
\gamma\left(k_{3}+E x^{0}\right) & B(\alpha-\beta / 2) \eta^{2}+B(\alpha+\beta / 2) \eta^{\dagger^{2}} \\
B(\alpha+\beta / 2) \eta^{2}+B(\alpha-\beta / 2) \eta^{\dagger^{2}} & -\gamma\left(k_{3}+E x^{0}\right)
\end{array}\right)\left(\begin{array}{l}
\chi_{\uparrow} \\
\chi_{\downarrow}
\end{array}\right) \\
& \equiv H_{R}(\gamma)\left(\begin{array}{c}
\chi_{\uparrow} \\
\chi_{\downarrow}
\end{array}\right) .
\end{aligned}
$$

The equation of motion for the field $\psi_{L}(x)$ can be obtained from equation (6.2) by means of the substitution $\gamma \rightarrow-\gamma$. In particular $H_{L}(\gamma)=H_{R}(-\gamma)$ in such a way that for every eigenfunction $\psi_{R}(x, \gamma)$ of the hamiltonian $H_{R}$, there exists a corresponding eigenfunction $\psi_{L}(x, \gamma)=\psi_{R}(x,-\gamma)$ of the hamiltonian $H_{L}$.

\subsection{Normalizable zero-energy modes}

In order to search for the chiral gapless Landau level which characterize the spectrum of $H_{R}\left(k_{3}\right)$, we must determine the zero-energy eigenstates of eq. (6.6). In particular, following Nielsen and Ninomiya, we consider the static problem with $E=0$ and we assume $\alpha \neq \pm \beta / 2$. Since the $\gamma$-term in $H_{R}$ anticommutes with the $\alpha$ and $\beta$ contribution, to search for a zero-energy solution we must impose $k_{3}=0$. Therefore we must look for normalized solutions $\chi_{0}$ of the equation:

$$
\left(\begin{array}{cc}
0 & B(\alpha-\beta / 2) \eta^{2}+B(\alpha+\beta / 2) \eta^{\dagger^{2}} \\
B(\alpha+\beta / 2) \eta^{2}+B(\alpha-\beta / 2) \eta^{\dagger^{2}} & 0
\end{array}\right)\left(\begin{array}{c}
\chi_{0, \uparrow} \\
\chi_{0, \downarrow}
\end{array}\right)=0 .
$$


Note that this equation is valid for both right $\psi_{R}(x)$ and left $\psi_{L}(x)$ field components, as long as $k_{3}=E=0$. This equation can be recast into the following relations for the components $\chi_{0, \uparrow}$ and $\chi_{0, \downarrow}$ :

$$
\begin{aligned}
& {\left[(\alpha-\beta / 2) \eta^{2}+(\alpha+\beta / 2) \eta^{\dagger^{2}}\right] \chi_{0, \downarrow}\left(\alpha, \beta, x_{1}\right)=0,} \\
& {\left[(\alpha+\beta / 2) \eta^{2}+(\alpha-\beta / 2) \eta^{\dagger^{2}}\right] \chi_{0, \uparrow}\left(\alpha, \beta, x_{1}\right)=0 .}
\end{aligned}
$$

These equations show that the two components are independent on each other, however we will show in the following that, given an arbitrary choice of $\alpha$ and $\beta$, only one of them can be normalized at a time. From the previous equations we also deduce that, if $\chi_{0, \uparrow}\left(\alpha, \beta, x_{1}\right)$ is a solution of eq. (6.9), $\chi_{0, \downarrow}\left(\alpha, \beta, x_{1}\right)=\chi_{0, \uparrow}\left(\alpha,-\beta, x_{1}\right)$ will be a solution of eq. (6.8), therefore we can limit our research to eq. (6.9) without loss of generality.

To solve equation (6.9), let us consider the basis provided by the wavefunctions of the harmonic oscillator $h_{n}\left(x_{1}\right)=\left(\eta^{\dagger}\right)^{n} h_{0}\left(x_{1}\right) / \sqrt{n !}$ : the operator in eq. (6.9) allows for transitions between $h_{n}$ and $h_{n \pm 2}$ only and the operator $(\alpha-\beta / 2) \eta^{2}$ annihilates both $h_{0}$ and $n_{1}$. The wavefunctions $h_{2}$ and $h_{3}$, instead, cannot be annihilated by the operator in eq. (6.9) and they must be absent from $\chi_{0, \uparrow}$. We deduce that there are two possible solutions of eq. (6.9) given by suitable linear combinations of the wavefunctions $h_{4 n}$ and $h_{4 n+1}$ respectively:

$$
\begin{aligned}
& \chi_{0, \uparrow}^{(1)}\left(x_{1}\right)=\sum_{n=0}^{\infty}(-1)^{n} d_{n}\left(\frac{\eta^{\dagger}}{2}\right)^{4 n} h_{0}\left(x_{1}\right), \\
& \chi_{0, \uparrow}^{(2)}\left(x_{1}\right)=\sum_{n=0}^{\infty}(-1)^{n} e_{n}\left(\frac{\eta^{\dagger}}{2}\right)^{4 n} \eta^{\dagger} h_{0}\left(x_{1}\right),
\end{aligned}
$$

where $d_{n}$ and $e_{n}$ are real coefficients. Equation (6.9) for the wavefunctions $\chi_{0, \uparrow}^{(i)}$ is fulfilled when:

$$
\begin{aligned}
\frac{d_{n+1}}{d_{n}} & =\left(\frac{\alpha-\beta / 2}{\alpha+\beta / 2}\right) \frac{1}{(n+1)(n+3 / 4)}, \\
\frac{e_{n+1}}{e_{n}} & =\left(\frac{\alpha-\beta / 2}{\alpha+\beta / 2}\right) \frac{1}{(n+5 / 4)(n+3 / 2)} .
\end{aligned}
$$

Both functions $\chi_{0, \uparrow}^{(i)}$ are normalizable if $\alpha \beta>0$. Indeed, one has for instance:

$$
\int d x_{1}\left|\chi_{0, \uparrow}^{(1)}\left(x_{1}\right)\right|^{2}=\sum_{n}\left|d_{n}\right|^{2}\left(\frac{1}{4}\right)^{4 n}(4 n) !
$$

The sum (6.12) is convergent since the large $n$ behaviour of the ratio of two consecutive addenda is given by

$$
\lim _{n \rightarrow \infty}\left[\left|d_{n+1}\right|^{2}\left(\frac{1}{4}\right)^{4(n+1)}[4(n+1)] !\right]\left[\left|d_{n}\right|^{2}\left(\frac{1}{4}\right)^{4 n}(4 n) !\right]^{-1}=\left(\frac{\alpha-\beta / 2}{\alpha+\beta / 2}\right)^{2},
$$

and when $\alpha \beta>0$ one has $[(\alpha-\beta / 2) /(\alpha+\beta / 2)]^{2}<1$. Thus $\chi_{0, \uparrow}^{(1)}$ has finite norm. Similarly, one easily shows that $\chi_{0, \uparrow}^{(2)}$ is normalizable as well. Moreover, the functions $\chi_{0, \uparrow}^{(1)}\left(x_{1}\right)$ and 
$\chi_{0, \uparrow}^{(2)}\left(x_{1}\right)$ are orthogonal, because they are obtained by applying even or odd numbers of creation operators $\eta^{\dagger}$ respectively on the ground state wavefunction $h_{0}\left(x_{1}\right)$. The previous functions, however, are not normalizable for $\alpha \beta<0$; this implies that, when the wavefunctions $\chi_{0, \uparrow}^{(i)}(\alpha, \beta)$ are well-defined, the corresponding wavefunctions $\chi_{0, \downarrow}^{(i)}(\alpha, \beta)=\chi_{0, \uparrow}^{(i)}(\alpha,-\beta)$ are not. We conclude that, when $\alpha \beta>0$ there are only two independent zero-energy modes given by:

$$
\chi_{0}^{(1)}=\left(\begin{array}{c}
\chi_{0, \uparrow}^{(1)}\left(x_{1}\right) \\
0
\end{array}\right), \quad \chi_{0}^{(2)}=\left(\begin{array}{c}
\chi_{0, \uparrow}^{(2)}\left(x_{1}\right) \\
0
\end{array}\right), \quad(\text { if } \alpha \beta>0),
$$

which satisfy equation (6.7). When $\alpha \beta<0$, instead, the role of $\chi_{0, \uparrow}$ and $\chi_{0, \downarrow}$ is exchanged. Two normalizable functions $\chi_{0, \downarrow}$ can be obtained from eq. (6.9). In this case the component $\chi_{0, \downarrow}$ can be described by two series of the kind (6.10) by imposing:

$$
\begin{aligned}
& \frac{d_{n+1}}{d_{n}}=\left(\frac{\alpha+\beta / 2}{\alpha-\beta / 2}\right) \frac{1}{(n+1)(n+3 / 4)} \\
& \frac{e_{n+1}}{e_{n}}=\left(\frac{\alpha+\beta / 2}{\alpha-\beta / 2}\right) \frac{1}{(n+5 / 4)(n+3 / 2)}
\end{aligned}
$$

for $\alpha \beta<0$, the convergence of these series is verified because $[(\alpha+\beta / 2) /(\alpha-\beta / 2)]^{2}<1$ and the zero-energy modes result

$$
\chi_{0}^{(1)}=\left(\begin{array}{c}
0 \\
\chi_{0, \downarrow}^{(1)}\left(x_{1}\right)
\end{array}\right), \quad \chi_{0}^{(2)}=\left(\begin{array}{c}
0 \\
\chi_{0, \downarrow}^{(2)}\left(x_{1}\right)
\end{array}\right), \quad(\text { if } \alpha \beta<0),
$$

with $\chi_{0, \downarrow}^{(i)}(\alpha,-\beta)=\chi_{0, \uparrow}^{(i)}(\alpha, \beta)$, relating the components in eqs. (6.14) and (6.16).

The result in [48] for the particular case with rotational invariance, for $\alpha= \pm \beta / 2$,

can be recovered observing that in these cases only the first term in each series for $\chi_{0}^{(i)}$ is different from zero.

\subsection{Dirac sea and the axial anomaly}

So far we discussed the zero-energy case. Now we reintroduce the $\gamma$ term in (6.6) and we assume $k_{3}, E \neq 0$. Let us consider the case $\alpha \beta>0$. The resulting chiral modes are of the form:

$$
\psi_{R / L}^{(i)}(x)=e^{i k_{2} x^{2}} e^{i k_{3} x^{3}} \chi_{0}^{(i)}\left(x^{1}\right) f_{R / L}\left(x^{0}\right),
$$

with $\chi_{0}^{(i)}\left(x^{1}\right)$ defined in equation (6.14) and the phase $f_{R / L}\left(x^{0}\right)$ to be determined by solving the Schrodinger equation (6.6): $i \partial_{0} \psi_{R / L}\left(x^{0}\right)=H_{R / L}\left(x^{0}\right) \psi_{R / L}\left(x^{0}\right)$. This equation can be solved by considering that $\chi_{0}^{(i)}$ has only one component which is annihilated by the offdiagonal terms of $H_{R / L}$, whereas the time-dependent diagonal term implies:

$$
\begin{aligned}
& f_{R}\left(x^{0}\right)=\exp \left[-i \gamma\left(k_{3} x^{0}+\frac{E\left(x^{0}\right)^{2}}{2}\right)\right], \\
& f_{L}\left(x^{0}\right)=\exp \left[i \gamma\left(k_{3} x^{0}+\frac{E\left(x^{0}\right)^{2}}{2}\right)\right], \quad(\text { if } \alpha \beta>0) .
\end{aligned}
$$


In the case $\alpha \beta<0$, instead, the wavefunctions $\chi_{0}^{(i)}$ possess only the second component (see equation (6.16)) and the resulting time dependence is:

$$
\begin{aligned}
& f_{R}\left(x^{0}\right)=\exp \left[i \gamma\left(k_{3} x^{0}+\frac{E\left(x^{0}\right)^{2}}{2}\right)\right], \\
& f_{L}\left(x^{0}\right)=\exp \left[-i \gamma\left(k_{3} x^{0}+\frac{E\left(x^{0}\right)^{2}}{2}\right)\right], \quad(\text { if } \alpha \beta<0) .
\end{aligned}
$$

With the definition of $\omega$ given by $i \partial_{0} \psi(x)=\omega \psi(x)$, we derive:

$$
\omega_{R}=\gamma\left(k_{3}+E x^{0}\right), \quad \omega_{L}=-\gamma\left(k_{3}+E x^{0}\right), \quad(\text { if } \alpha \beta>0),
$$

and

$$
\omega_{R}=-\gamma\left(k_{3}+E x^{0}\right), \quad \omega_{L}=\gamma\left(k_{3}+E x^{0}\right), \quad(\text { if } \alpha \beta<0) .
$$

These equations are consistent with a constant acceleration of the particles along $x^{3}$ given by $\partial_{0} \omega$ and define the chiral nature of these gapless modes, reflecting the linear dispersion as a function of $k_{3}$. Vacuum stability requires that the values of $\omega_{R}$ and $\omega_{L}$ must be nonnegative. Therefore, in agreement with the Dirac sea interpretation of the fermions ground state, the stable vacuum of the system corresponds to the state in which all the single-particle states with negative frequencies are occupied. All the other Landau levels $\psi_{R}(x)$ and $\psi_{L}(x)$, which are orthogonal to the modes (6.17), are not chiral, namely they have frequencies with a symmetric dispersion for $k_{3} \rightarrow-k_{3}$ and they never cross zero energy: during the time evolution, the sign of their frequency does not change. This implies that these gapped Landau levels are either totally empty or totally filled, and, when considering the effect of the acceleration caused by $E$, they do not contribute to the net rate of change of the right or left particle number [14]. Thus, as far as the axial anomaly is concerned, we only need to discuss the vacuum stability with respect to the modes (6.17).

Let us consider the case $\alpha \beta>0$ and $\gamma>0$. The value (6.20) of $\omega_{R}$ is negative for $k_{3}<-E x^{0}$. Therefore all the right-handed one-particle states with $k_{3}<-E x^{0}$ must be occupied, and the available states for a $\psi_{R}$ particle are only those with $k_{3}>-E x^{0}$. Similarly, since $\omega_{L}$ is negative for $k_{3}>-E x^{0}$, all the left-handed one-particle states with $k_{3}>-E x^{0}$ must be occupied and the available states for a $\psi_{L}$ particle are only those with $k_{3}<-E x^{0}$.

Let us recall that, if the one-particle states are labelled by the values $\boldsymbol{k}$ of the momentum, the number $N$ of available states for one particle moving inside a cubic box of volume $V=L^{3}$ is specified (in the large $V$ limit) by $d N=L^{3} d^{3} k /(2 \pi)^{3}$. Therefore, in our system the number $N_{R}$ of available $\psi_{R}$ states is determined by the product

$$
N_{R}=2 \times \text { Landau degeneracy } \times \text { range of } k_{3},
$$

where the factor 2 is due to the presence of the two modes $\chi_{0}^{(1)}$ and $\chi_{0}^{(2)}$. The Landau degeneracy is determined by the range of $k_{2}$ which guarantees the particle localisation inside the box, $0 \leq\left(x^{1}-k_{2} / B\right) \leq L$,

$$
\text { Landau degeneracy }=L \int_{0}^{B L} \frac{d k_{2}}{(2 \pi)}=\frac{B L^{2}}{2 \pi} .
$$


One gets

$$
N_{R}=2 \times L \int_{0}^{B L} \frac{d k_{2}}{(2 \pi)} \times L \int_{-E x^{0}}^{\infty} \frac{d k_{3}}{(2 \pi)}=\frac{2 B V}{(2 \pi)^{2}} \int_{-E x^{0}}^{\infty} d k_{3},
$$

and then

$$
\partial_{0} N_{R}=\frac{2 E B V}{4 \pi^{2}} .
$$

By means of the same argument, one determines the number $N_{L}$ of available $\psi_{L}$ states

$$
N_{L}=2 \times L \int_{0}^{B L} \frac{d k_{2}}{(2 \pi)} \times L \int_{-\infty}^{-E x^{0}} \frac{d k_{3}}{(2 \pi)}=\frac{2 B V}{(2 \pi)^{2}} \int_{-\infty}^{-E x^{0}} d k_{3}
$$

and thus

$$
\partial_{0} N_{L}=-\frac{2 E B V}{4 \pi^{2}}
$$

Consequently, one finds

$$
\int d^{3} x \partial_{0} J_{A}^{0}(x)=\partial_{0} N_{R}-\partial_{0} N_{L}=\frac{V 4 E B}{4 \pi^{2}}=-V \frac{2}{4 \pi^{2}} \epsilon^{\mu \nu \tau \lambda} \partial_{\mu} A_{\nu} \partial_{\tau} A_{\lambda},
$$

which is in agreement with the perturbative computation of the axial anomaly of section 5. It is now easy to verify that, for all the possible nontrivial values of $\alpha, \beta$ and $\gamma$, the axial anomaly computed by means of the Nielsen-Ninomiya method coincides with expression (3.9). This concludes the derivation of the result (3.9) by means of the NielsenNinomiya method.

Finally we observe that the vector current is conserved, since

$$
\int d^{3} x\left(\partial_{0} J_{R}^{0}(x)+\partial_{0} J_{L}^{0}(x)\right)=\partial_{0} N_{R}+\partial_{0} N_{L}=0
$$

The Nielsen-Ninomiya method suggests that the axial anomaly is stable against perturbations of the chemical potentials around the (zero) energy of the multi-Weyl nodes. We shall discuss this issue in section 11

\section{Atiyah-Singer index}

The axial anomaly can also be interpreted [55, 76-79] as the index of the euclidean analytic extension of the operator which acts on the fermion field in the expression (3.5) of the lagrangian. The index can be defined as the number of nontrivial normalizable solutions with zero eigenvalues of this lagrangian operator, having support in $\mathbb{R}^{4}$. Moreover, the null solutions for the right and left parts can be identified separately, and the index results from the difference of their numbers. By using the Atiyah-Singer approach, in this section we shall rederive the result (3.9). 


\subsection{Particles and antiparticles}

In the presence of the classical external electric $E$ and magnetic $B$ fields shown in equation (6.1), from the lagrangian (3.5) one can derive the equations of motion for the "righthanded" particle wave functions $\psi_{R}$,

$$
\left\{i \partial_{0}-\alpha\left[\partial_{1}^{2}-\left(\partial_{2}-i B x^{1}\right)^{2}\right] \sigma^{1}-\frac{\beta}{2}\left\{\partial_{1},\left(\partial_{2}-i B x^{1}\right)\right\} \sigma^{2}+i \gamma\left(\partial_{3}+i E x^{0}\right) \sigma^{3}\right\} \psi_{R}(x)=0,
$$

and for the "left-handed" particle wave functions $\psi_{L}$,

$$
\left\{i \partial_{0}-\alpha\left[\partial_{1}^{2}-\left(\partial_{2}-i B x^{1}\right)^{2}\right] \sigma^{1}-\frac{\beta}{2}\left\{\partial_{1},\left(\partial_{2}-i B x^{1}\right)\right\} \sigma^{2}-i \gamma\left(\partial_{3}+i E x^{0}\right) \sigma^{3}\right\} \psi_{L}(x)=0 .
$$

Let $\psi_{R}^{C}$ and $\psi_{L}^{C}$ represent the wave functions of the "right-handed" and "left-handed" antiparticles respectively,

$$
\psi_{R}^{C}=\left(\psi_{R}\right)^{C}=\left(-i \sigma^{2}\right) \psi_{R}^{*}(x) \quad, \quad \psi_{L}^{C}=\left(\psi_{L}\right)^{C}=\left(i \sigma^{2}\right) \psi_{L}^{*}(x) .
$$

The equations of motion for $\psi_{R}^{C}$ and $\psi_{L}^{C}$ in the given classical electromagnetic background (6.1) take the form

$$
\left\{-i \partial_{0}+\alpha\left[\partial_{1}^{2}-\left(\partial_{2}+i B x^{1}\right)^{2}\right] \sigma^{1}+\frac{\beta}{2}\left\{\partial_{1},\left(\partial_{2}+i B x^{1}\right)\right\} \sigma^{2}+i \gamma\left(\partial_{3}-i E x^{0}\right) \sigma^{3}\right\} \psi_{R}^{C}(x)=0,
$$

and

$$
\left\{-i \partial_{0}+\alpha\left[\partial_{1}^{2}-\left(\partial_{2}+i B x^{1}\right)^{2}\right] \sigma^{1}+\frac{\beta}{2}\left\{\partial_{1},\left(\partial_{2}+i B x^{1}\right)\right\} \sigma^{2}-i \gamma\left(\partial_{3}-i E x^{0}\right) \sigma^{3}\right\} \psi_{L}^{C}(x)=0 .
$$

The field operators $\psi_{R}$ and $\psi_{L}$ describe four kinds of particles: one "right-handed" particle and its antiparticle, and one "left-handed" particle and its antiparticle. The four relations (7.1), (7.2), (7.4) and (7.5) represent precisely a complete set of corresponding equations.

\subsection{Euclidean zero modes}

With fixed electromagnetic background, let us consider the analytic extension of the operators entering the equations of motion (6.1), (6.2), (6.4) and (6.6) in the euclidean region [80] (which is obtained by means of the replacement $p_{0} \rightarrow i p_{0}$ ). We need to determine [55] the corresponding normalizable zero modes in $\mathbb{R}^{4}$.

Let us examine the case $\alpha \beta>0$ and $\gamma>0$. One can specify the values of the two spatial components $p_{2}$ and $p_{3}$ of the momentum by putting

$$
\begin{aligned}
\psi_{R}(x) & =e^{i k_{2} x^{2}} e^{i k_{3} x^{3}} \tilde{\psi}_{R}\left(x^{1}, x^{0}\right), & \psi_{L}(x) & =e^{i k_{2} x^{2}} e^{i k_{3} x^{3}} \tilde{\psi}_{L}\left(x^{1}, x^{0}\right) \\
\psi_{R}^{C}(x) & =e^{-i k_{2} x^{2}} e^{-i k_{3} x^{3}} \tilde{\psi}_{R}^{C}\left(x^{1}, x^{0}\right), & & \psi_{L}^{C}(x)=e^{-i k_{2} x^{2}} e^{-i k_{3} x^{3}} \tilde{\psi}_{L}^{C}\left(x^{1}, x^{0}\right) .
\end{aligned}
$$


In addition to the $\eta$ and $\eta^{\dagger}$ operators defined in equation (6.4), it is useful to introduce the ladder operators $\zeta$ and $\zeta^{\dagger}$,

$$
\zeta=\frac{1}{\sqrt{2 \gamma E}} \partial_{0}+\sqrt{\frac{\gamma E}{2}}\left(x^{0}+\frac{k_{3}}{E}\right), \quad \zeta^{\dagger}=-\frac{1}{\sqrt{2 \gamma E}} \partial_{0}+\sqrt{\frac{\gamma E}{2}}\left(x^{0}+\frac{k_{3}}{E}\right),
$$

satisfying the canonical commutation relations $\left[\zeta, \zeta^{\dagger}\right]=1$. Let $f_{0}\left(x^{0}\right)$ be the normalised ground state wave function satisfying $\zeta f_{0}\left(x^{0}\right)=0$. The euclidean analytic extensions of equations (7.1), (7.2), (7.4) and (7.5) assume the form

$$
\begin{gathered}
\left\{-\sqrt{\frac{\gamma E}{2}}\left(\zeta-\zeta^{\dagger}\right)-\alpha B\left(\eta^{2}+\left(\eta^{\dagger}\right)^{2}\right) \sigma^{1}+i \frac{\beta}{2} B\left(\eta^{2}-\left(\eta^{\dagger}\right)^{2}\right) \sigma^{2}-\sqrt{\frac{\gamma E}{2}}\left(\zeta+\zeta^{\dagger}\right) \sigma^{3}\right\} \widetilde{\psi}_{R}=0, \\
\left\{-\sqrt{\frac{\gamma E}{2}}\left(\zeta-\zeta^{\dagger}\right)-\alpha B\left(\eta^{2}+\left(\eta^{\dagger}\right)^{2}\right) \sigma^{1}+i \frac{\beta}{2} B\left(\eta^{2}-\left(\eta^{\dagger}\right)^{2}\right) \sigma^{2}+\sqrt{\frac{\gamma E}{2}}\left(\zeta+\zeta^{\dagger}\right) \sigma^{3}\right\} \widetilde{\psi}_{L}=0, \\
\left\{\sqrt{\frac{\gamma E}{2}}\left(\zeta-\zeta^{\dagger}\right)+\alpha B\left(\eta^{2}+\left(\eta^{\dagger}\right)^{2}\right) \sigma^{1}+i \frac{\beta}{2} B\left(\eta^{2}-\left(\eta^{\dagger}\right)^{2}\right) \sigma^{2}+\sqrt{\frac{\gamma E}{2}}\left(\zeta+\zeta^{\dagger}\right) \sigma^{3}\right\} \widetilde{\psi}_{R}^{C}=0, \\
\left\{\sqrt{\frac{\gamma E}{2}}\left(\zeta-\zeta^{\dagger}\right)+\alpha B\left(\eta^{2}+\left(\eta^{\dagger}\right)^{2}\right) \sigma^{1}+i \frac{\beta}{2} B\left(\eta^{2}-\left(\eta^{\dagger}\right)^{2}\right) \sigma^{2}-\sqrt{\frac{\gamma E}{2}}\left(\zeta+\zeta^{\dagger}\right) \sigma^{3}\right\} \widetilde{\psi}_{L}^{C}=0 .
\end{gathered}
$$

The complete set of normalizable solutions in $\mathbb{R}^{4}$ of equations (7.8)-(7.11) can easily be determined because these equations depend on separated variable, since $\eta$ and $\eta^{\dagger}$ commute with $\zeta$ and $\zeta^{\dagger}$. Equations (7.9) and (7.10) do not admit normalizable solutions. Whereas equation (7.8) admits the normalizable solutions

$$
\widetilde{\psi}_{R}\left(x^{1}, x^{0}\right)=\left(\begin{array}{c}
\chi_{0, \uparrow}^{(i)}\left(x^{1}\right) f_{0}\left(x^{0}\right) \\
0
\end{array}\right), \quad \text { with } i=1,2,
$$

and equation (7.11) admits the normalizable solutions

$$
\widetilde{\psi}_{L}^{C}\left(x^{1}, x^{0}\right)=\left(\begin{array}{c}
0 \\
\chi_{0, \uparrow}^{(i)}\left(x^{1}\right) f_{0}\left(x^{0}\right)
\end{array}\right), \quad \text { with } i=1,2,
$$

where the functions $\chi_{0, \uparrow}^{(i)}$ are defined in equations (6.10) and we are exploiting the mapping between right and left sectors. By exploiting the separability of the Landau level structures defined by the $\zeta$ and $\eta$ operators, we can map the previous wavefunction in a $4 \mathrm{D}$ quantum Hall problem [81, 82]. We obtain that inside a hypercube in $\mathbb{R}^{4}$ of hypervolume $V_{4}=L^{4}$ the Landau degeneracy of the each of the zero modes (7.12) and (7.13) is given by

$$
\text { Landau degeneracy }=L \int_{0}^{B L} \frac{d k_{2}}{(2 \pi)} \times L \int_{0}^{L E} \frac{d k_{3}}{(2 \pi)}=\frac{E B L^{4}}{4 \pi^{2}} .
$$


Therefore the number $\nu_{R}$ of euclidean zero modes, which are associated with the "righthanded particles", is given by

$$
\nu_{R}=2 \times \frac{E B L^{4}}{4 \pi^{2}},
$$

and the number $\bar{\nu}_{L}$ of euclidean zero modes, which are associated with the "left-handed antiparticles", is found to be

$$
\bar{\nu}_{L}=2 \times \frac{E B L^{4}}{4 \pi^{2}},
$$

Therefore the index of the euclidean extension of the lagrangian operator - acting on the fermion fields - turns out to be

$$
\frac{\nu_{R}+\bar{\nu}_{L}}{V_{4}}=4 \times \frac{E B}{4 \pi^{2}}=-\frac{2}{4 \pi^{2}} \epsilon^{\mu \nu \tau \lambda} \partial_{\mu} A_{\nu} \partial_{\tau} A_{\lambda},
$$

which is in agreement with the expression (3.9) of the axial anomaly. One can easily verify that this agreement still holds for arbitrary values of $\alpha, \beta$ and $\gamma$. This concludes the rederivation of the result (3.9) by means of the Atiyah-Singer index argument.

We note that the approach followed in the present section suggests a direct relation between the lagrangian zero modes and the chiral states derived from the corresponding hamiltonians in section 6 , allowing for a parallelism between the two related methods to obtain the chiral anomalies.

\section{Axial anomaly for triple-Weyl semimetals}

In this section, the axial anomaly for the triple-Weyl semimetals model is derived by means of the Nielsen-Ninomiya and the Atiyah-Singer arguments because, in this case, the perturbative quantum field theory procedure requires considerable effort.

As it has been discussed in section 6 , in order to implement the Nielsen-Ninomiya procedure we need to consider the equations of motion which are derived from the lagrangian (3.11) in the presence of the gauge fields background (6.1). For the moment, let us consider the case in which $\gamma>0$. The analogues of equations (6.6) take the form

$$
i \partial_{0}\left(\begin{array}{c}
\chi_{\uparrow} \\
\chi_{\downarrow}
\end{array}\right)=\left(\begin{array}{cc}
\gamma\left(k_{3}+E x^{0}\right) & B(\alpha-\beta) \eta^{3}+B(\alpha+\beta) \eta^{\dagger^{3}} \\
B(\alpha+\beta) \eta^{3}+B(\alpha-\beta) \eta^{\dagger^{3}} & -\gamma\left(k_{3}+E x^{0}\right)
\end{array}\right)\left(\begin{array}{l}
\chi_{\uparrow} \\
\chi_{\downarrow}
\end{array}\right),
$$

Therefore, we search again zero-energy $(\omega=0)$ normalizable solutions of the equation for $\gamma=0$ :

$$
\left(\begin{array}{cc}
0 & c(\alpha-\beta) \eta^{3}+B(\alpha+\beta) \eta^{\dagger^{3}} \\
B(\alpha+\beta) \eta^{3}+B(\alpha-\beta) \eta^{\dagger^{3}} & 0
\end{array}\right)\left(\begin{array}{l}
\chi_{\uparrow} \\
\chi_{\downarrow}
\end{array}\right)=0 .
$$

For this purpose, similarly to equation (6.10), we introduce the following ansatz:

$$
\left(\begin{array}{c}
\chi_{\uparrow} \\
\chi_{\downarrow}
\end{array}\right)=\left(\begin{array}{c}
\sum_{n} a_{n} h_{n}\left(x^{1}\right) \\
\sum_{n} b_{n} h_{n}\left(x^{1}\right)
\end{array}\right)
$$


where $h_{n}\left(x^{1}\right)$ are the wavefunction of the $n$-th Landau level. By inserting this expansion in eq. (8.2) we obtain, for $n \geq 3$ :

$$
\begin{aligned}
& (\alpha+\beta) b_{n-3} K_{n-3,+}+(\alpha-\beta) b_{n+3} K_{n+3,-}=0 \\
& (\alpha-\beta) a_{n-3} K_{n-3,+}+(\alpha+\beta) a_{n+3} K_{n+3,-}=0
\end{aligned}
$$

where $K_{n+3,-}=\sqrt{(n+1)(n+2)(n+3)}$ and $K_{n-3,+}=\sqrt{n(n-1)(n-2)}$. We impose $b_{n-3}=K_{n-3,+}=0$ if $n<3$.

The two equations (8.4) and (8.5) decouple. Therefore, by following the same argument presented in section 6 after equation (6.13), we conclude that normalizable solutions with $\omega=0$ exist only if $\left\{a_{n}\right\}=0$ or $\left\{b_{n}\right\}=0$. By direct inspection, we find that $\left\{a_{n}\right\}=$ 0 if $\alpha \beta<0$, while $\left\{b_{n}\right\}=0$ if $\alpha \beta>0$. In each case, we obtain three independent normalizable solutions with $\omega=0$, corresponding to values $\left\{a_{0}, a_{1}, a_{2}\right\}$ or $\left\{b_{0}, b_{1}, b_{2}\right\}$ to be fixed. Following the same arguments of the section 6 one obtains:

$$
\int d^{3} x \partial_{0} J_{A}^{0}(x)=\partial_{0} N_{R}-\partial_{0} N_{L}=\frac{3 E B V}{4 \pi^{2}}-\left(-\frac{3 E B V}{4 \pi^{2}}\right)=\frac{V 6 E B}{4 \pi^{2}}=-V \frac{3}{4 \pi^{2}} \epsilon^{\mu \nu \tau \lambda} \partial_{\mu} A_{\nu} \partial_{\tau} A_{\lambda},
$$

which is in agreement with equation (3.12). One can easily verify that a modification of the signs of the coefficients $\alpha, \beta$ and $\gamma$ is taken into account by the $\Theta(\alpha, \beta, \gamma)$ factor defined in expression (3.10).

The derivation of the axial anomaly by means of the Atiyah-Singer argument is quite simple. Indeed, the counting of the euclidean zero modes is carried out by means of two steps:

- (by adopting the notations of section 7), the $\zeta, \zeta^{\dagger}$ part in the $\mathbb{R}^{4}$ euclidean space gives a factor 1 , precisely as the case of the double-Weyl model;

- whereas the $\eta, \eta^{\dagger}$ part gives a multiplicative factor 3 , because there are three independent normalizable solutions of equation (8.2) with $\mathcal{E}=0$.

Consequently, in agreement with equation (8.6), the axial anomaly of the triple-Weyl model reads

$$
\partial_{\mu} J_{A}^{\mu}(x)=-3 \Theta(\alpha, \beta, \gamma) \frac{1}{4 \pi^{2}} \epsilon^{\mu \nu \tau \lambda} \partial_{\mu} A_{\nu}(x) \partial_{\tau} A_{\lambda}(x) .
$$

This concludes the proof of equation (3.12).

\section{Triple-point semimetals model}

The lagrangian of the triple-point semimetals model is shown in equation (3.13). In order to compute the axial anomaly, we shall first use the Nielsen-Ninomiya method, and then the Atiyah-Singer argument, because the standard perturbative approach suffers from difficulties due to the existence of singular points in the parameter space. Let us consider the fermionic fields in the presence of the gauge background

$$
A_{0}(x)=0, \quad A_{1}(x)=0, \quad A_{2}(x)=-B x^{1}, \quad A_{3}(x)=E x^{0},
$$


with $E>0$ and $B>0$. Since the gauge background does not depend on $x^{2}$ and $x^{3}$, one can specify the values $k_{2}$ and $k_{3}$ of the components $p_{2}$ and $p_{3}$ of the momentum

$$
\psi_{R}(x)=e^{i k_{2} x^{2}} e^{i k_{3} x^{3}} \chi\left(x^{0}, x^{1}\right), \quad \psi_{L}(x)=e^{i k_{2} x^{2}} e^{i k_{3} x^{3}} \xi\left(x^{0}, x^{1}\right),
$$

and the equations of motion following from the lagrangian (3.13) take the form

$$
\begin{aligned}
i \partial_{0} \chi & =i v\left[M_{1} \partial_{1}+M_{2}\left(i k_{2}-i B x^{1}\right)+M_{3}\left(i k_{3}+i E x^{0}\right)\right] \chi \\
i \partial_{0} \xi & =i v\left[M_{1} \partial_{1}+M_{2}\left(i k_{2}-i B x^{1}\right)-M_{3}\left(i k_{3}+i E x^{0}\right)\right] \xi .
\end{aligned}
$$

Let us recall that we need to determine [14] the crossing rate of the energy eigenvalues of the single particle states through the zero level. Since the (linear) time dependence of the hamiltonian is contained in the covariant derivative $D_{3}$ exclusively, it is convenient to introduce the two normalised eigenvectors $\Sigma_{ \pm}$of $M_{3}$ with nontrivial eigenvalues,

$$
\Sigma_{ \pm}=\frac{1}{\sqrt{2}}\left(\begin{array}{c}
\mp e^{i \theta / 2} \\
i e^{-i \theta / 2} \\
0
\end{array}\right), \quad M_{3} \Sigma_{ \pm}= \pm \Sigma_{ \pm}
$$

A normalizable (in the $x^{1}$ variable) zero eigenvector $u_{0}\left(x^{1}\right)$ of the "reduced hamiltonian" iv $\left(M_{1} D_{1}+M_{2} D_{2}\right)$ must satisfy the equation

$$
\left(M_{1} D_{1}+M_{2} D_{2}\right) u_{0}=\left(\begin{array}{ccc}
0 & 0 & e^{-i \theta}\left(B x^{1}-k_{2}\right) \\
0 & 0 & e^{i \theta}\left(i \partial_{1}\right) \\
-e^{i \theta}\left(B x^{1}-k_{2}\right) & e^{-i \theta}\left(-i \partial_{1}\right) & 0
\end{array}\right) u_{0}\left(x^{1}\right)=0 .
$$

The normalizable solutions of equation (9.4) are given by

$$
\begin{array}{llll}
u_{0}\left(x^{1}\right)=\Sigma_{+} \exp \left[-\frac{1}{2 B}\left(B x^{1}-k_{2}\right)^{2} e^{i 3 \theta}\right], & \text { when } & \cos (3 \theta)>0, \\
u_{0}\left(x^{1}\right)=\Sigma_{-} \exp \left[\frac{1}{2 B}\left(B x^{1}-k_{2}\right)^{2} e^{i 3 \theta}\right], & \text { when } & \cos (3 \theta)<0 .
\end{array}
$$

In the case $\cos (3 \theta)=0$, equation (9.4) does not admit normalizable solutions. We point out that this condition corresponds to the condition $\sin (3 \theta)=0$ in the notation of [50]. The fermionic modes

$$
\psi_{R}(x)=e^{i k_{2} x^{2}} e^{i k_{3} x^{3}} u_{0}\left(x^{1}\right) f_{R}\left(x^{0}\right), \quad \psi_{L}(x)=e^{i k_{2} x^{2}} e^{i k_{3} x^{3}} u_{0}\left(x^{1}\right) f_{L}\left(x^{0}\right),
$$

satisfy the equation $i \partial_{0} \psi(x)=\omega \psi(x)$, (then $f_{R / L}\left(x^{0}\right)$ defined similarly as in (6.18) and (6.19)), with frequencies

$$
\omega_{R}=-v\left(k_{3}+E x^{0}\right), \quad \omega_{L}=v\left(k_{3}+E x^{0}\right), \quad(\text { if } \cos (3 \theta)>0),
$$

and

$$
\omega_{R}=v\left(k_{3}+E x^{0}\right), \quad \omega_{L}=-v\left(k_{3}+E x^{0}\right), \quad(\text { if } \cos (3 \theta)<0) .
$$


During the time evolution, the values of these frequencies crosses the zero value; thus the modes (9.6) contribute to the axial anomaly. While the remaining fermionic modes have frequencies with fixed signs and can be neglected [14]. Therefore, when $\cos (3 \theta)>0$, for particles moving inside a cubic box of volume $V=L^{3}$, the numbers $N_{R}$ and $N_{L}$ of available one particle states are given by

$$
N_{R}=L \int_{0}^{B L} \frac{d k_{2}}{(2 \pi)} \times L \int_{-\infty}^{-E x^{0}} \frac{d k_{3}}{(2 \pi)}, \quad N_{L}=L \int_{0}^{B L} \frac{d k_{2}}{(2 \pi)} \times L \int_{-E x^{0}}^{\infty} \frac{d k_{3}}{(2 \pi)} .
$$

Hence the vector gauge symmetry is not anomalous

$$
\partial_{0} N_{R}+\partial_{0} N_{L}=0
$$

whereas

$$
\int d^{3} x \partial_{0} J_{A}^{0}(x)=\partial_{0} N_{R}-\partial_{0} N_{L}=-\frac{V 2 E B}{4 \pi^{2}}=V \frac{1}{4 \pi^{2}} \epsilon^{\mu \nu \tau \lambda} \partial_{\mu} A_{\nu} \partial_{\tau} A_{\lambda} .
$$

Similarly, when $\cos (3 \theta)<0$, one finds

$$
\int d^{3} x \partial_{0} J_{A}^{0}(x)=\partial_{0} N_{R}-\partial_{0} N_{L}=+\frac{V 2 E B}{4 \pi^{2}}=-V \frac{1}{4 \pi^{2}} \epsilon^{\mu \nu \tau \lambda} \partial_{\mu} A_{\nu} \partial_{\tau} A_{\lambda} .
$$

As illustrated in section 7 , the computation of the axial anomaly by means of the Atiyah-Singer approach is strictly connected with the Nielsen-Ninomiya method. Indeed, in the presence of the gauge background (6.1), the number of euclidean zero modes can be written as the product of the Landau degeneracy (7.14) with the number of the normalised zero modes of the "reduced hamiltonian" of equation (9.4). Therefore, also for the triplepoint model one can easily verify that the Atiyah-Singer argument leads to a result in complete agreement with equations (9.11) and (9.12).

To sum up, in the case of the triple-point semimetals model with lagrangian (3.13), when the vector gauge invariance is maintained, the axial anomaly is given by

$$
\partial_{\mu} J_{A}^{\mu}(x)=\frac{\cos (3 \theta)}{|\cos (3 \theta)|} \frac{1}{4 \pi^{2}} \epsilon^{\mu \nu \tau \lambda} \partial_{\mu} A_{\nu}(x) \partial_{\tau} A_{\lambda}(x) .
$$

This concludes the derivation of equation (3.16). Let us recall that the axial anomaly (9.13) has been obtained for vanishing chemical potential; in our notations, this means that all the one-particle states with zero energy are assumed to be occupied. A discussion on the stability of the result (9.13) against perturbations of the chemical potentials is contained in section 11, as well as on its relation with the presence of semi-chiral states with asymptotically vanishing energy $[50,83]$. These semi-chiral states could also contribute to the normalization of the vector current associated to the chiral magnetic effect $[13,84]$, which is strictly related to the axial anomaly.

\section{Quantization of the anomaly coefficient}

In section 4 , it has been mentioned that the overall multiplicative factor $\mathcal{N}$, which appears in equation (4.10), can only assume integer values. According to the interpretations of 
Nielsen-Ninomiya and Atiyah-Singer of the axial anomaly [14, 55, 56], the quantisation of $\mathcal{N}$ naturally emerges. We would like to present here another argument - confirming the quantisation of $\mathcal{N}$ - which is based on perturbation theory, and which is of interest because it makes use of the relationship between the abelian and the nonabelian gauge anomalies [71-73, 85]. In the case of non-abelian anomalies, the quantisation of the anomaly normalization factor has been discussed for instance in [86-89].

Let us briefly recall where the emergence of the chiral gauge anomaly is found in perturbation theory. Suppose that the lagrangian for a fermion field $\psi_{R}(x)$ in the presence of a classical gauge potential $V_{\mu}(x)$ takes the form

$$
\mathcal{L}_{R}=\psi_{R}^{\dagger}(x) \Pi_{R}(V) \psi_{R}(x)
$$

in which $\Pi_{R}(V)$ represents a certain differential operator which is a function of the covariant derivatives $D_{\mu}=\partial_{\mu}+i V_{\mu}$. As we have seen in the previous sections, the field $\psi_{R}(x)$ may contain several components, and not necessarily it represents a spinor field. Let us assume that $\mathcal{L}_{R}$ is invariant under local gauge transformations $\psi_{R}(x) \rightarrow e^{i \theta_{R}(x)} \psi_{R}(x)$, $V_{\mu}(x) \rightarrow V_{\mu}(x)-\partial_{\mu} \theta_{R}(x)$. The renormalized sum of the connected one-loop vacuum-tovacuum diagrams of the $\psi_{R}(x)$ field - in the presence of the classical background $V_{\mu}(x)$ - is denoted by $i \Gamma_{R}[V]$. Here it is assumed that $\Gamma_{R}[V]$ admits a perturbative expansion in powers of the background gauge field $V_{\mu}(x)$. Despite the gauge invariance of $\mathcal{L}_{R}$, the infinitesimal gauge variation of $\Gamma_{R}[V]$ may be nonvanishing and, modulo the variation of local counterterms, it is given by

$$
\delta_{\theta_{R}} \Gamma_{R}[V]=-\mathcal{N} \frac{1}{24 \pi^{2}} \int d^{4} x \epsilon^{\mu \nu \tau \lambda} \partial_{\mu} \theta_{R}(x) V_{\nu}(x) \partial_{\tau} V_{\lambda}(x)
$$

When $\mathcal{N} \neq 0$, the classical gauge invariance $V_{\mu}(x) \rightarrow V_{\mu}(x)-\partial_{\mu} \theta_{R}(x)$ is broken by the presence of an anomaly. We have already mentioned the universality of the local function $\epsilon^{\mu \nu \tau \lambda} \partial_{\mu} V_{\nu} \partial_{\tau} V_{\lambda}$ entering expression (10.2). We would like to elaborate now on the quantisation of $\mathcal{N}$.

Let us consider the chiral anomaly in the case of a non-abelian gauge symmetry. The field theory model defined by the lagrangian (10.1) will now be modified in order to introduce a non-abelian symmetry. Suppose that a certain field theory model contains $N$ (with $N>2$ ) copies of the fermion field $\psi_{R}(x)$. Thus the variables of this new model can be described by the fields $\psi_{R}^{j}(x)$, where the index $j$, that we call the flavour index, takes values $j=1,2 \ldots, N$; this set of fields will be denoted by $\Psi_{R}(x)$. An internal symmetry group acts on the $N$ components $\psi_{R}^{j}(x)$ of $\Psi_{R}(x)$ according to the fundamental representation of $\mathrm{SU}(N)_{R}$. Let now the gauge field $V_{\mu}(x)$ take values in the Lie algebra of $\mathrm{SU}(N)_{R}$, $V_{\mu}(x)=V_{\mu}^{a}(x) T^{a}$, where $\left\{T^{a}\right\}$ (with $a=1,2, \ldots, N^{2}-1$ ) are the generators of $\mathrm{SU}(N)_{R}$. Let the lagrangian of the model be

$$
\mathcal{L}_{R}=\Psi_{R}^{\dagger}(x) \Pi_{R}(V) \Psi_{R}(x),
$$

where, in the differential operator $\Pi_{R}(V)$, the abelian covariant derivative $D_{\mu}=\partial_{\mu}+i V_{\mu}(x)$ has been replaced by the non-abelian covariant derivative $\left(D_{\mu}\right)_{j k}=\delta_{j k} \partial_{\mu}+i V_{\mu}^{a}(x) T_{j k}^{a}$, and 
a sum over all the flavour indices is understood. The lagrangian (10.3) is invariant under $\mathrm{SU}(N)_{R}$ gauge transformations. Under an infinitesimal $\mathrm{SU}(N)_{R}$ gauge transformation $V_{\mu}(x) \rightarrow V_{\mu}(x)-\partial_{\mu} \theta_{R}(x)+i\left[\theta_{R}(x), V_{\mu}(x)\right]$, the gauge variation of the renormalized oneloop functional $\Gamma_{R}^{\prime}[V]$ of the nonabelian model is given by

$$
\delta_{\theta_{R}} \Gamma_{R}^{\prime}[V]=-\mathcal{N} \frac{1}{24 \pi^{2}} \int d^{4} x \epsilon^{\mu \nu \tau \lambda} \operatorname{Tr}\left[\partial_{\mu} \theta_{R}(x)\left(V_{\nu}(x) \partial_{\tau} V_{\lambda}(x)+(i / 2) V_{\mu}(x) V_{\nu}(x) V_{\lambda}(x)\right)\right] .
$$

The fields polynomial which must be integrated in expression (10.4) satisfies [7] the WessZumino consistency conditions. It is important to note that the multiplicative coefficient $\mathcal{N}$ that appears in equation (10.4) is exactly the same coefficient $\mathcal{N}$ entering equation (10.2). This equality is well known in the context of the computation [71, 72] of the chiral anomalies by means of perturbation theory. Indeed, in the perturbative computation of the anomaly (10.4), the Feynman diagrams which contribute to the term of expression (10.4) which is quadratic in $V_{\mu}(x)$ precisely coincide with the diagrams which enter the computation of the abelian anomaly (10.2). In the perturbative computation of this term, the non commutativity of the $\mathrm{SU}(N)$ generators is harmless because the field combination $\epsilon^{\mu \nu \tau \lambda} \partial_{\mu} V_{\nu} \partial_{\tau} V_{\lambda}$ is symmetric under the exchange $\partial_{\mu} V_{\nu} \leftrightarrow \partial_{\tau} V_{\lambda}$. As far as these Feynman diagrams are concerned, the only difference between the abelian and the nonabelian case is that, in the nonabelian case, in the end of the computations one has to take a sum over the flavour indices, or, one needs to introduce a trace over the indices of the fundamental $\mathrm{SU}(N)_{R}$ representation. Note that the presence of this trace is explicitly indicated in expression (10.4). Therefore the same Feynman diagrams which produce the coefficient $\mathcal{N}$ in equation (10.2) necessarily yield the same coefficient $\mathcal{N}$ in equation (10.4).

At this point, in order to complete the argument, we need to show that the coefficient $\mathcal{N}$ multiplying the nonabelian anomaly (9.4) must take integer values. Instead of displaying a formal proof, let us produce a physical argument.

The relationship of the abelian chiral anomaly (4.10) and the corresponding abelian axial anomaly (4.21) has been discussed in section 4; let us consider the non-abelian generalisation of this relationship. In the nonabelian case, suppose that, in addition to the field $\Psi_{R}(x)$, one also has the fermionic field $\Psi_{L}(x)$ made of $N$ components $\psi_{L}^{j}(x)$, with $j=1,2, \ldots, N$ and the corresponding lagrangian term is

$$
\mathcal{L}_{L}=\Psi_{L}^{\dagger}(x) \Pi_{L}(W) \Psi_{L}(x) .
$$

The differential operator $\Pi_{L}(W)$ is a function of the covariant derivative $\left(D_{\mu}\right)_{j k}=\delta_{j k} \partial_{\mu}+$ $i W_{\mu}^{a}(x) T_{j k}^{a}$, where $W_{\mu}(x)=W_{\mu}^{a}(x) T^{a}$ is the connection of the gauge group $\mathrm{SU}(N)_{L}$, which acts on the components $\psi_{L}^{j}(x)$. It is assumed that the lagrangian (10.5) is invariant under $\mathrm{SU}(N)_{L}$ gauge transformations, with $\Psi_{L}(x)$ transforming according to the fundamental $\mathrm{SU}(N)_{L}$ representation. Let us assume that the chiral $\mathrm{SU}(N)_{L}$ anomaly takes the form

$$
\delta_{\theta_{L}} \Gamma_{L}^{\prime}[W]=\mathcal{N} \frac{1}{24 \pi^{2}} \int d^{4} x \epsilon^{\mu \nu \tau \lambda} \operatorname{Tr}\left[\partial_{\mu} \theta_{L}(x)\left(W_{\nu}(x) \partial_{\tau} W_{\lambda}(x)+(i / 2) W_{\mu}(x) W_{\nu}(x) W_{\lambda}(x)\right)\right]
$$


where the multiplicative factor $\mathcal{N}$ is the same factor $\mathcal{N}$ appearing in expression (10.4). This is precisely what one finds with fermion multiplets (as quarks and leptons fields) of ordinary spinor fields $\Psi_{R}(x)$ and $\Psi_{L}(x)$ minimally coupled with gauge fields.

In the composed field theory model which contains both the $N$-components field $\Psi_{R}(x)$ and the $N$-components field $\Psi_{L}(x)$ and total lagrangian

$$
\mathcal{L}=\Psi_{R}^{\dagger}(x) \Pi_{R}(V) \Psi_{R}(x)+\Psi_{L}^{\dagger}(x) \Pi_{L}(W) \Psi_{L}(x),
$$

where $V_{\mu}(x)$ and $W_{\mu}(x)$ are classical background fields, one has an anomalous flavour symmetry group $\mathrm{SU}(N)_{R} \times \mathrm{SU}(N)_{L}$. The $\mathrm{SU}(N)_{R} \times \mathrm{SU}(N)_{L}$ gauge variation of the one-loop functional $\Gamma^{\prime}[V, W]=\Gamma_{R}^{\prime}[V]+\Gamma_{L}^{\prime}[W]$ is given by the sum of expressions (10.4) and (10.6). Similarly to the abelian case, by adding a suitable finite local Bardeen counterterm $L_{B}[V, W]$ to the one-loop functional $\Gamma^{\prime}[V, W]$, one can define [71] a new functional $\Gamma[V, W]=\Gamma^{\prime}[V, W]+L_{B}[V, W]$ which is invariant under transformations of the vector subgroup $\mathrm{SU}(N)_{V}$ of $\mathrm{SU}(N)_{R} \times \mathrm{SU}(N)_{L}$. Only axial transformations (with infinitesimal parameters given by the difference $\left.\theta_{R}-\theta_{L}\right)$ are anomalous. The resulting Bardeen flavour anomaly [71] of the axial component of the group $\mathrm{SU}(N)_{R} \times \mathrm{SU}(N)_{L}$ is proportional to $\mathcal{N}$.

When this flavour anomaly is integrated [7] by employing for instance the so-called "Goldstone bosons" field [90, 91] $\mathrm{U}(x) \in \mathrm{SU}(N)$, the corresponding Wess-Zumino term is proportional to $\mathcal{N}$. The Wess-Zumino term is well defined [7, 92, 93] — and the ambiguities which are originated by the obstruction given by the non triviality of $\pi_{5}(\mathrm{SU}(N))$ are harmless - only when $\mathcal{N} \in \mathbb{Z}$. On the other hand, the Wess-Zumino term must be well defined because, in the case of the low energy effective lagrangian [90, 91] of the hadrons physics in which $N=3$, for instance, it describes part of the hadronic interactions of the light pseudo scalar mesons of the octet - and part of the interactions between these mesons and the flavour gauge fields of the Standard Model — which can be observed in laboratory. A few consequences of the quantisation of $\mathcal{N}$ in particles physics can also be found, for instance, in references [86-89, 94-102]. Therefore the value of the multiplicative factor $\mathcal{N}$, entering expressions (10.6), (10.4) and (10.2) must be an integer.

The quantization of the anomaly multiplicative factor $\mathcal{N}$ has nontrivial consequences in the field theory models in which the fermion lagrangian terms contain free parameters, as in the case of the parameters $\{\alpha, \beta, \gamma\}$ in the multi-Weyl models (3.5) and (3.11). Indeed, since any smooth variation of a quantised coefficient must be vanishing, in these models the expression of the axial anomaly must be invariant under "smooth" variations of the parameters, i.e. "smooth" modification of the operator acting on the fermion fields in the lagrangian. And in facts, this is precisely the outcome of the explicit computations of the axial anomalies (3.9) and (3.12), which depend on $\{\alpha, \beta, \gamma\}$ through the variable

$$
\Theta(\alpha, \beta, \gamma)=\frac{\alpha \beta \gamma}{|\alpha \beta \gamma|}
$$

The function $\Theta(\alpha, \beta, \gamma)$ is locally constant. All the modifications of the value of $\Theta(\alpha, \beta, \gamma)$ are found when one of the parameters $\{\alpha, \beta, \gamma\}$ changes its sign; that is, when the value of one of the parameters crosses the zero point. Note that the zero value of one of these parameters represents a critical point for the lagrangian operators $\Pi_{R}(V)$ or $\Pi_{L}(W)$ entering 
the lagrangian. Indeed, when $\alpha=0$ or $\beta=0$, for instance, there are no more normalizable solutions of equations (6.7) and (8.2). Consequently, a modification of the sign of one of the parameters $\{\alpha, \beta, \gamma\}$ does not corresponds to a "smooth" modification of the operators $\Pi_{R}(V)$ or $\Pi_{L}(W)$.

Similarly, in the case of the triple-point semimetals model (3.13) the dependence of the axial anomaly (3.16) on the $\theta$ parameter is given by the multiplicative factor

$$
\frac{\cos (3 \theta)}{|\cos (3 \theta)|}
$$

which is locally constant. The change of sign of this factor occurs for $3 \theta= \pm \pi / 2$, which correspond to critical points for the operators appearing in the lagrangian (3.13). Indeed, as it has been shown in section 9 , when $3 \theta= \pm \pi / 2$ the zero eigenvectors of the reduced hamiltonian (9.4) are not normalizable.

A consequence of the quantization of the anomaly is that, if a term $\delta E(\mathbf{k}) \propto$ $q k_{x}\left(\psi_{R}^{\dagger}(\mathbf{k}) \psi_{R}(\mathbf{k}) \pm \psi_{L}^{\dagger}(\mathbf{k}) \psi_{L}(\mathbf{k})\right)$ (terms proportional to $\propto q k_{y}$ or $\propto q k_{z}$ are possible as well) is added to the Weyl lagrangian (3.1), no variation of the anomaly from the form (3.4) is obtained, until $q$ is strong enough to induce a transition to a type-II Weyl-semimetal [103]. In the latter condition, the Fermi surface becomes extended and important deviations in the anomaly are expected. We predict the same situation for type-II generalizations of doubleand triple-Weyl semimetals, driven by terms as $\delta E(\mathbf{k}) \propto q k_{x}^{l}\left(\psi_{R}^{\dagger}(\mathbf{k}) \psi_{R}(\mathbf{k}) \pm \psi_{L}^{\dagger}(\mathbf{k}) \psi_{L}(\mathbf{k})\right)$, with $l=2,3$ respectively.

\section{Chemical potentials and stability}

The computations of the axial anomaly that have been presented in the previous sections refer to the case in which all the single-particle states with negative energy are occupied. This corresponds to the situation in which the chemical potential coincides with the energy of the band-touching nodes of the semimetals. Let us now consider the stability of our results under modifications of the chemical potentials.

\subsection{Multi-Weyl semimetals}

In the perturbative approach, a discussion on the stability of the chiral gauge anomalies for a single Weyl model can also be found, for instance, in the paper [71] by Bardeen, in which the most general bilinear couplings of the fermions with external sources have been considered. Actually, the stability of the axial anomaly has a general validity that we shall now examine.

As it is shown in equations (3.5), (3.11) and (3.13), the lagrangian density $\mathcal{L}$ of the various models that we have considered in the present article has the common structure

$$
\mathcal{L}=\Psi^{\dagger}(x)\left\{i D_{0}-\mathcal{H}(\boldsymbol{D})\right\} \Psi(x)=\Psi^{\dagger}(x)\left\{i\left(\partial_{0}+i A_{0}\right)-\mathcal{H}(\boldsymbol{D})\right\} \Psi(x)
$$

where $\Psi=\left(\psi_{R}, \psi_{L}\right)$ and $\mathcal{H}(\boldsymbol{D})$ represents a differential operator constructed with the spatial components of the covariant derivative $\boldsymbol{D}_{j}=\partial_{j}+i A_{j}(x)$, with $j=1,2,3$. The 
modification of the chemical potentials can be described by the introduction of the additional lagrangian term

$$
\Delta \mathcal{L}=\mu_{R} \psi_{R}^{\dagger}(x) \psi_{R}(x)+\mu_{L} \psi_{L}^{\dagger}(x) \psi_{L}(x)
$$

where $\mu_{R}$ and $\mu_{L}$ are constant parameters. For sufficiently small values of $\mu_{R}$ and $\mu_{L}$, does the addition of $\Delta \mathcal{L} \neq 0$ modify the expression of the axial anomaly? In other words, is the axial anomaly, computed for the model which is described by the lagrangian $\mathcal{L}+\Delta \mathcal{L}$, equal to the axial anomaly which is found for the model with lagrangian $\mathcal{L}$ ?

When $\mu_{R}=\mu_{L}=\mu$, the modified lagrangian density $\mathcal{L}+\Delta \mathcal{L}$ takes the form

$$
\mathcal{L}+\Delta \mathcal{L}=\Psi^{\dagger}\left\{i\left[\partial_{0}+i\left(A_{0}-\mu\right)\right]-\mathcal{H}(\boldsymbol{D})\right\} \Psi
$$

which is equal to expression (11.1) with the only replacement $A_{0}(x) \rightarrow A_{0}(x)-\mu$. The axial anomaly expression $\epsilon^{\mu \nu \tau \lambda} \partial_{\mu} A_{\nu}(x) \partial_{\tau} A_{\lambda}(x)$ is not modified by the replacement $A_{0}(x) \rightarrow$ $A_{0}(x)-\mu$. Therefore the introduction of the chemical potential $\mu \neq 0$ does not alter the expression of the axial anomaly. The stability of the axial anomaly if $\mu_{R}=\mu_{L}$ is suggested directly also by the Nielsen-Ninomiya method. Indeed, the addition of a term $\mu \psi^{\dagger}(x) \psi(x)$ modifies the zero-point of the energy spectrum but, in the presence of electric and magnetic fields, it does not modify the crossing rate of the energy values through the zero level. Therefore, the introduction of $\mu$ would simply amount to a constant energy shift $\omega \rightarrow \omega+\mu$ which does not affect the values of the rates $\partial_{0} N_{R}$ and $\partial_{0} N_{L}$ of equations (6.25) and (6.27).

When $\mu_{L}=-\mu_{R}=\mu_{5}$, the lagrangian density $\mathcal{L}+\Delta \mathcal{L}$ takes the form

$$
\mathcal{L}+\Delta \mathcal{L}=\Psi^{\dagger}\left\{i\left[\partial_{0}+i A_{0}+i \mu_{5} \gamma^{5}\right]-\mathcal{H}(\boldsymbol{D})\right\} \Psi
$$

where $\gamma^{5}=\operatorname{diag}(1,-1)$. This expression describes the lagrangian of a model in which the fermion variable $\Psi(x)$ is coupled with the gauge connection $A_{\mu}$ of the group $\mathrm{U}(1)_{V}$ in the usual way, and $\Psi(x)$ is also coupled with the gauge connection $B_{\mu}(x)$ of the group $\mathrm{U}(1)_{A}$ in which $B_{0}(x)=\mu_{5}$ and $\boldsymbol{B}(x)=0$. As it has been demonstrated in section 4 , in this case, when the $\mathrm{U}(1)_{V}$ gauge invariance is preserved, the axial anomaly is given by expression (4.20)

$$
\delta_{\theta_{A}} \Gamma[A, B]=\mathcal{N} \frac{1}{12 \pi^{2}} \int d^{4} x \epsilon^{\mu \nu \tau \lambda} \theta_{A}(x)\left[\partial_{\mu} B_{\nu}(x) \partial_{\tau} B_{\lambda}(x)+3 \partial_{\mu} A_{\nu}(x) \partial_{\tau} A_{\lambda}(x)\right] .
$$

This equation shows that the contribution of the $B_{\mu}$ field to the axial anomaly is vanishing when $B_{0}(x)=0$ and $\boldsymbol{B}(x)=0$, and it is vanishing also when $B_{0}(x)=\mu_{5}$ and $\boldsymbol{B}(x)=0$. Therefore, the introduction of the chemical potential $\mu_{5} \neq 0$ also does not modify the expression of the axial anomaly. Note that the presence of $\mu_{5} \neq 0$ gives rise to the chiral magnetic effect $[13,84]$, which is connected to the axial anomaly and is described by the vector current

$$
j_{\nu}=-\mathcal{N} \mu_{5} \frac{e^{2}}{2 \pi^{2}} \epsilon^{0 \nu \alpha \beta} \partial_{\alpha} A_{\beta}
$$




\subsection{Triple-point semimetals}

The computation of the axial anomaly and the stability of the corresponding result in the case of the triple-point semimetal deserves a particular discussion. Let us recall that the lagrangians of the type shown in equations (3.5), (3.11) and (3.13) represent phenomenological approximations of more complicated theories which describe the dynamics of the relevant fermionic degrees of freedom in the various materials. Usually, the validity of these simplified expressions is limited to a neighbourhood of the locations of the touching nodes in the Brillouin zone; in our notations, these neighbourhoods correspond to the low momenta regions. The study of the effective models, which are defined by the phenomenological lagrangians, may by useful because, in certain cases, one can easily deduce interesting features which are common to both these low-energy models and to the true physical systems. The computation of the axial anomaly is precisely one example of this strategy, in which it is assumed that the axial anomaly of the effective theories coincides with the axial anomaly of the corresponding real systems. However, it turns out that the low-energy model associated with the triple-point semimetal, which is defined by the lagrangian (3.13), presents certain unrealistic peculiarities that must be taken into account in order to determine the axial anomaly.

In the case of vanishing gauge potential, $A_{\mu}(x)=0$, the energy spectrum of the oneparticle states, which is defined by the lagrangian (3.13) when $\theta=0$, for instance, contains a flat band with vanishing energy, such that $\omega(\boldsymbol{k})=0$, for all values of $\boldsymbol{k}$. As a consequence, standard perturbation theory - in which one makes an expansion of the Feynman diagrams in powers of the classical fields $A_{\mu}(x)$ - cannot be defined for the theory (3.13), because the Feynman propagator does not exist. The existence of a flat band of this type, with arbitrarily large momentum and zero energy, is usually unstable under realistic perturbations of the Hamiltonian and can be considered as an unphysical artifact of the model.

Although the Feynman diagrams approach cannot be utilised, in order to determine the axial anomaly, one can still use the Nielsen-Ninomiya method (or, equivalently, the Atiyah-Singer approach), as it has been illustrated in section 9. But also in this case one finds certain unphysical features that must be taken into account. Indeed, as it has been shown in $[50,83]$, in the presence of a magnetic field directed, for instance, along the $x^{3}$ direction, in addition to the chiral states with wave functions (9.6), other two Landau levels of normalizable states (here quoted "semi-chiral") emerge, which have zero energy only asymptotically $\left(k_{3} \rightarrow \pm \infty\right)$; for fixed chirality, in appropriate simplified notations, the corresponding dispersion relations have the hyperbolic form $\widetilde{\omega}^{( \pm)}\left(k_{3}\right)=k_{3} \pm \sqrt{k_{3}^{2}+B^{2}}$. The branch $\widetilde{\omega}^{(+)}\left(k_{3}\right)$ describes particle states with decreasing and vanishing energy as $k_{3} \rightarrow-\infty$, whereas $\widetilde{\omega}^{(-)}\left(k_{3}\right)$ corresponds to antiparticle (or hole) states with decreasing and vanishing energy as $k_{3} \rightarrow \infty$.

The asymptotic behaviour of $\widetilde{\omega}^{( \pm)}\left(k_{3}\right)$ in the large $\left|k_{3}\right|$ limit appears to be rather unreliable. Indeed, for realistic lattice (tight-binding) models, it is likely that the semichiral states display a modified dispersion at momenta sufficiently far from the nodes, and a finite separation $\Delta$ in energy from the zero level, due at least to the finite extension of the Brillouin zone. Moreover, these semi-chiral states could be subject to a strong mixing 


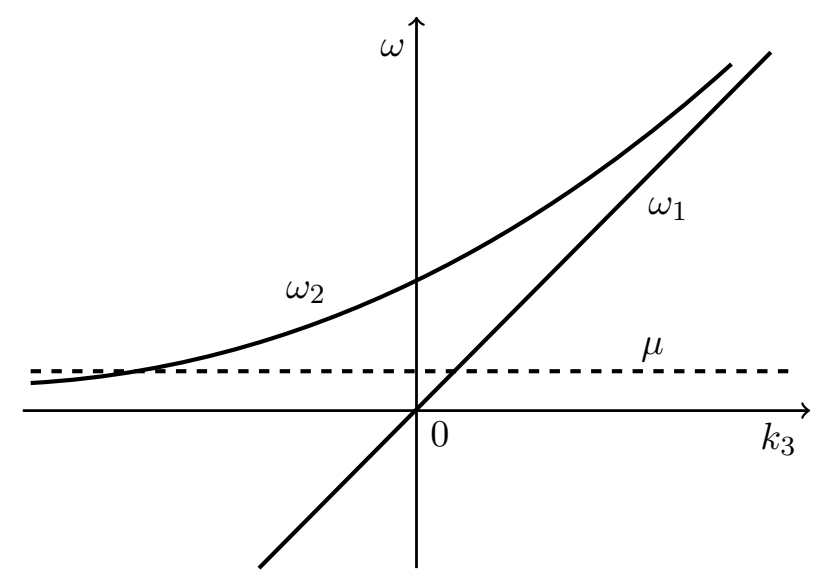

Figure 2. Energy levels $\omega_{1}\left(k_{3}\right)$ and $\omega_{2}\left(k_{3}\right)$ for single-particle states, obtained from the effective theory of equation (3.13).

effect due to the magnetic field. Their peculiar dispersion which approaches zero energy at large momenta $k_{3}$ can indeed favor the coupling of the hyperbolic branches belonging to triple-point crossings with opposite chiralities and we reckon the consequent mixing effect to be stronger than the one for Weyl semimetals [104]. In this scenario, the semi-chiral modes would develop a finite energy gap, separating them from the flat band.

Let us now concentrate on the two chiral Landau levels that appear for positive energies and are relevant for the computation of the axial anomaly. The first has a dispersion relation $\omega_{1}\left(k_{3}\right)$ linear in $k_{3}$ (in appropriate notations one can put $\omega_{1}\left(k_{3}\right)=2 k_{3}$ ), the wave functions of the corresponding states are shown in equation (9.6). The second has instead a dispersion relation of the hyperbolic form $\omega_{2}\left(k_{3}\right)=\widetilde{\omega}^{(+)}\left(k_{3}\right)=k_{3}+\sqrt{k_{3}^{2}+B^{2}}$. The behaviours of $\omega_{1}\left(k_{3}\right)$ and $\omega_{2}\left(k_{3}\right)$ are sketched in figure 2 .

For vanishing chemical potential, only the branch $\omega_{1}\left(k_{3}\right)$ intersects the zero energy level and, according to the Nielsen-Ninomiya procedure, it gives a unitary contribution to the integer coefficient $\mathcal{N}$ appearing in the axial anomaly. However, as it is shown in figure 2 , both energy branches $\omega_{1}\left(k_{3}\right)$ and $\omega_{2}\left(k_{3}\right)$ intersect the energy level determined by a nonvanishing value $\mu>0$ of the chemical potential. So, one could claim that, in this case, the multiplicative integer coefficient of the axial anomaly must be doubled, $|\mathcal{N}|=2$ (with a negative value of the chemical potential, one needs to consider the branch $\widetilde{\omega}^{(-)}\left(k_{3}\right)$ instead of $\widetilde{\omega}^{(+)}\left(k_{3}\right)$, getting the same conclusion). However, the value of $k_{3}$ corresponding to the intersection point of $\omega_{2}\left(k_{3}\right)$ with the $\mu$ energy level tends to $-\infty$ as $\mu$ approaches to zero. Thus, for sufficiently small $\mu$, the intersection point is outside the validity range of the lowenergy effective theory (3.13), and the lattice corrections mentioned above should no more be neglected. This is why, in the computation of the axial anomaly for the triple-point semimetals presented in section 9, we have chosen to take into account of the dispersion relation $\omega_{1}\left(k_{3}\right)$ exclusively, thus modeling the behavior for $\mu$ approaching zero.

Summing up, we expect that our result $|\mathcal{N}|=1$ for the axial anomaly coefficient of the triple-point semimetals is stable under sufficiently small modifications of the chemical potentials from zero energy. If the mixing of the semi-chiral modes due to the magnetic 
field is not strong enough to develop a gap, by varying the chemical potential of the system, we possibly expect to find a transition from a phase displaying $|\mathcal{N}|=1$ for small $\mu$, to a phase with $|\mathcal{N}|=2$ for larger values of it.

\section{Conclusions}

In this article we have derived the expression of the abelian axial anomaly for the doubleWeyl, triple-Weyl and triple-point semimetal models. Three different computation methods have been considered: the perturbative quantum field theory procedure which is based on the evaluation of the one-loop Feynman diagrams, the Nielsen-Ninomiya method, and the Atiyah-Singer index argument. The consistency of these methods, which have been shown to be closely related, has been illustrated in detail in the case of the double-Weyl model. For the triple-Weyl and the triple-point models the perturbative approach is rather burdensome or affected by singularities; therefore only the Nielsen-Ninomiya and Atiyah-Singer methods have been discussed. It has been shown that the dependence of the anomaly on the vector gauge field $A_{\mu}(x)$ is not contingent on the Lorentz symmetry, but is determined by the gauge symmetry structure. In facts, the axial anomaly takes the general form

$$
\partial_{\mu} J_{A}^{\mu}=\mathcal{N} \frac{1}{16 \pi^{2}} F \wedge F
$$

where $F=\left(\partial_{\mu} A_{\nu}-\partial_{\nu} A_{\mu}\right) d x^{\mu} \wedge d x^{\nu}$ denotes the curvature 2-form, and the value of the multiplicative factor $\mathcal{N}$ is determined by the lagrangian of each model. General arguments, still based on gauge invariance, suggest that the factor $\mathcal{N}$ must be quantized and must match the topological charge of the corresponding band touching points. Indeed, this is precisely the outcome of the explicit anomaly computations. We have found that $|\mathcal{N}|=2$ for the double-Weyl model, $|\mathcal{N}|=3$ for the triple-Weyl model and $|\mathcal{N}|=1$ for the triple-point model. The last result has been obtained by neglecting the hyperbolic Landau levels with asymptotical vanishing energy in the limit of large momenta. Indeed, we have presented arguments supporting this choice. For this reason, our result is not in contradiction with the usual counting of the chiral states in the triple-point semimetals presented in $[50,83])$. We have further discussed the stability of the anomaly under smooth modifications of the lagrangian parameters, showing that the value of $\mathcal{N}$ is invariant under these deformations. The modification of the sign of $\mathcal{N}$ in the considered models has been examined. We have verified that, in the parameter space, the points in which the value of $\mathcal{N}$ undergoes a change of sign indeed correspond to critical points. Finally we have shown that, in agreement with the case of a single-Weyl model, a modification of the chemical potentials does not change the expression of the axial anomaly.

\section{Acknowledgments}

It is a great pleasure to thank Ion Cosma Fulga, Massimo Mannarelli, Michele Mintchev, Giampiero Paffuti, Simone Paganelli, and Andrea Trombettoni for useful discussions. 
Open Access. This article is distributed under the terms of the Creative Commons Attribution License (CC-BY 4.0), which permits any use, distribution and reproduction in any medium, provided the original author(s) and source are credited.

\section{References}

[1] C. Itzykson and J.-B. Zuber, Quantum field theory, McGraw-Hill, U.S.A., (1980) [INSPIRE].

[2] S. Weinberg, The quantum theory of fields, vol. 1, Cambridge University Press, Cambridge, U.K., (1995) [INSPIRE].

[3] M.E. Peskin and D.V. Schroeder, An introduction to quantum field theory, Addison-Wesley, Reading, MA, U.S.A., (1995) [INSPIRE].

[4] J.S. Schwinger, On gauge invariance and vacuum polarization, Phys. Rev. 82 (1951) 664 [INSPIRE].

[5] S.L. Adler, Axial vector vertex in spinor electrodynamics, Phys. Rev. 177 (1969) 2426 [INSPIRE].

[6] J.S. Bell and R. Jackiw, A PCAC puzzle: $\pi^{0} \rightarrow \gamma \gamma$ in the $\sigma$-model, Nuovo Cim. A 60 (1969) 47 [INSPIRE].

[7] J. Wess and B. Zumino, Consequences of anomalous Ward identities, Phys. Lett. B 37 (1971) 95 [INSPIRE].

[8] B. Zumino, Cohomology of gauge groups: cocycles and Schwinger terms, Nucl. Phys. B 253 (1985) 477 [INSPIRE].

[9] T. Hatsuda and T. Kunihiro, QCD phenomenology based on a chiral effective Lagrangian, Phys. Rept. 247 (1994) 221 [hep-ph/9401310] [INSPIRE].

[10] M.Z. Hasan and C.L. Kane, Topological insulators, Rev. Mod. Phys. 82 (2010) 3045 [arXiv: 1002.3895] [INSPIRE].

[11] X.-L. Qi and S.-C. Zhang, Topological insulators and superconductors, Rev. Mod. Phys. 83 (2011) 1057 [arXiv: 1008. 2026] [INSPIRE].

[12] S. Ryu, J.E. Moore and A.W.W. Ludwig, Electromagnetic and gravitational responses and anomalies in topological insulators and superconductors, Phys. Rev. B 85 (2012) 045104 [arXiv: 1010.0936] [INSPIRE].

[13] A.A. Zyuzin and A.A. Burkov, Topological response in Weyl semimetals and the chiral anomaly, Phys. Rev. B 86 (2012) 115133 [arXiv:1206.1868] [INSPIRE].

[14] H.B. Nielsen and M. Ninomiya, Adler-Bell-Jackiw anomaly and Weyl fermions in crystal, Phys. Lett. B 130 (1983) 389 [INSPIRE].

[15] B.Q. Lv et al., Experimental discovery of Weyl semimetal TaAs, Phys. Rev. X 5 (2015) 031013.

[16] B.Q. Lv et al., Observation of Weyl nodes in TaAs, Nature Phys. 11 (2015) 724 [arXiv: 1503.09188].

[17] S.-Y. Xu et al., Discovery of a Weyl fermion semimetal and topological Fermi arcs, Science 349 (2015) 613 [INSPIRE].

[18] S.-Y. Xu et al., Discovery of a Weyl fermion semimetal state in NbAs, Nature Phys. 11 (2015) 748 [arXiv:1504.01350]. 
[19] X. Wan, A.M. Turner, A. Vishwanath and S.Y. Savrasov, Topological semimetal and Fermi-arc surface states in the electronic structure of pyrochlore iridates, Phys. Rev. B 83 (2011) 205101 [INSPIRE].

[20] A.A. Burkov and L. Balents, Weyl semimetal in a topological insulator multilayer, Phys. Rev. Lett. 107 (2011) 127205 [arXiv: 1105.5138] [INSPIRE].

[21] S.A. Parameswaran, T. Grover, D.A. Abanin, D.A. Pesin and A. Vishwanath, Probing the chiral anomaly with nonlocal transport in three-dimensional topological semimetals, Phys. Rev. X 4 (2014) 031035.

[22] J. Xiong et al., Evidence for the chiral anomaly in the Dirac semimetal $\mathrm{Na}_{3} B i$, Science $\mathbf{3 5 0}$ (2015) 413.

[23] X. Huang et al., Observation of the chiral-anomaly-induced negative magnetoresistance in $3 D$ Weyl semimetal TaAs, Phys. Rev. X 5 (2015) 031023.

[24] C. Zhang et al., Signatures of the Adler-Bell-Jackiw chiral anomaly in a Weyl fermion semimetal, Nature Commun. 7 (2016) 10735.

[25] Q. Li et al., Chiral magnetic effect in ZrTe $e_{5}$, Nature Phys. 12 (2016) 550.

[26] H.B. Nielsen and M. Ninomiya, No go theorem for regularizing chiral fermions, Phys. Lett. B 105 (1981) 219 [inSPIRE].

[27] L. Lepori, I.C. Fulga, A. Trombettoni and M. Burrello, PT invariant Weyl semimetals in gauge symmetric systems, Phys. Rev. B 94 (2016) 085107 [arXiv:1506.04761] [INSPIRE].

[28] H.J. Rothe, Lattice gauge theories: an introduction, World Sci. Lect. Notes Phys. 82 (2005) 1 [INSPIRE].

[29] G.E. Volovik, The universe in a helium droplet, Oxford University Press, Oxford, U.K., (2003).

[30] S.-Y. Xu et al., Observation of Fermi arc surface states in a topological metal, Science $\mathbf{3 4 7}$ (2015) 294.

[31] N. Xu et al., Observation of Weyl nodes and Fermi arcs in tantalum phosphide, Nature Commun. 7 (2016) 11006.

[32] L. Lu et al., Experimental observation of Weyl points, Science 349 (2015) 622.

[33] W.-J. Chen, M. Xiao and C.T. Chan, Photonic crystals possessing multiple Weyl points and the experimental observation of robust surface states, Nature Commun. 7 (2016) 13038.

[34] J. Noh, S. Huang, D. Leykam, Y.D. Chong, K.P. Chen and M.C. Rechtsman, Experimental observation of optical Weyl points and Fermi arc-like surface states, Nature Phys. 13 (2017) 611.

[35] F. Li, X. Huang, J. Lu, J. Ma and Z. Liu, Weyl points and Fermi arcs in a chiral phononic crystal, Nature Phys. 14 (2018) 30.

[36] C. Fang, M.J. Gilbert, X. Dai and B.A. Bernevig, Multi-Weyl topological semimetals stabilized by point group symmetry, Phys. Rev. Lett. 108 (2012) 266802.

[37] G. Xu, H. Weng, Z. Wang, X. Dai and Z. Fang, Chern semi-metal and quantized anomalous Hall effect in $\mathrm{HgCr}_{2} \mathrm{Se}_{4}$, Phys. Rev. Lett. 107 (2011) 186806 [arXiv:1106.3125] [INSPIRE].

[38] V. Shivamoggi and M.J. Gilbert, Weyl phases in point-group symmetric superconductors, Phys. Rev. B 88 (2013) 134504. 
[39] S.-K. Jian and H. Yao, Correlated double-Weyl semimetals with Coulomb interactions: possible applications to $\mathrm{HgCr}_{2} \mathrm{Se}_{4}$ and $\mathrm{SrSi}_{2}$, Phys. Rev. B 92 (2015) 045121.

[40] X. Dai, H.-Z. Lu, S.-Q. Shen and H. Yao, Detecting monopole charge in Weyl semimetals via quantum interference transport, Phys. Rev. B 93 (2016) 161110.

[41] Q. Chen and G.A. Fiete, Thermoelectric transport in double-Weyl semimetals, Phys. Rev. B 93 (2016) 155125.

[42] S.M. Huang et al., New type of Weyl semimetal with quadratic double Weyl fermions, Proc. Natl. Acad. Sci. 113 (2016) 1180.

[43] L. Lepori, I.C. Fulga, A. Trombettoni and M. Burrello, Double Weyl points and Fermi arcs of topological semimetals in non-Abelian gauge potentials, Phys. Rev. A 94 (2016) 053633 [arXiv: 1603.02292] [INSPIRE].

[44] X.-Y. Mai, D.-W. Zhang, Z. Li and S.-L. Zhu, Exploring topological double-Weyl semimetals with cold atoms in optical lattices, Phys. Rev. A 95 (2017) 063616 [arXiv:1706.02063] [INSPIRE].

[45] L.-J. Lang, S.-L. Zhang, K.T. Law and Q. Zhou, Weyl points and topological nodal superfluids in a face-centered-cubic optical lattice, Phys. Rev. B 96 (2017) 035145 [arXiv: 1611.08671] [INSPIRE].

[46] W.-J. Chen, M. Xiao and C.T. Chan, Photonic crystals possessing multiple Weyl points and the experimental observation of robust surface states, Nature Commun. 7 (2016) 13038.

[47] B. Roy and J.D. Sau, Magnetic catalysis and axionic charge-density-wave in Weyl semimetals, Phys. Rev. B 92 (2015) 125141 [arXiv:1406.4501] [INSPIRE].

[48] X. Li, B. Roy and S. Das Sarma, Weyl fermions with arbitrary monopoles in magnetic fields: Landau levels, longitudinal magnetotransport and density-wave ordering, Phys. Rev. B 94 (2016) 195144 [arXiv: 1608. 06632] [INSPIRE].

[49] Z.-M. Huang, J. Zhou and S.-Q. Shen, Topological responses from chiral anomaly in multi-Weyl semimetals, Phys. Rev. B 96 (2017) 085201 [arXiv:1705.04576] [INSPIRE].

[50] B. Bradlyn et al., Beyond Dirac and Weyl fermions: unconventional quasiparticles in conventional crystals, Science 353 (2016) 558.

[51] Z. Zhu, G.W. Winkler, Q. Wu, J. Li and A.A. Soluyanov, Triple point topological metals, Phys. Rev. X 6 (2016) 031003.

[52] I.C. Fulga and A. Stern, Triple point fermions in a minimal symmorphic model, Phys. Rev. B 95 (2017) 241116 [arXiv: 1702.03939] [INSPIRE].

[53] H. Hu, J. Hou, F. Zhang and C. Zhang, Topological triply-degenerate points induced by spin-tensor-momentum couplings, arXiv:1709.04964.

[54] B.Q. Lv et al., Observation of three-component fermions in the topological semimetal molybdenum phosphide, Nature 546 (2017) 627.

[55] M.F. Atiyah and I.M. Singer, Dirac operators coupled to vector potentials, Proc. Natl. Acad. Sci. 81 (1984) 2597.

[56] M. Nakahara, Geometry, topology and physics, $2^{\text {nd }}$ edition, Institute of Physics, Bristol, U.K., (2003).

[57] C.-K. Chiu, J.C.Y. Teo, A.P. Schnyder and S. Ryu, Classification of topological quantum matter with symmetries, Rev. Mod. Phys. 88 (2016) 035005. 
[58] N.P. Armitage, E.J. Mele and A. Vishwanath, Weyl and Dirac semimetals in three-dimensional solids, Rev. Mod. Phys. 90 (2018) 015001.

[59] I.C. Fulga, L. Fallani and M. Burrello, Geometrically protected triple-point crossings in an optical lattice, Phys. Rev. B 97 (2018) 121402 [arXiv:1711.10935] [INSPIRE].

[60] D.T. Son and N. Yamamoto, Berry curvature, triangle anomalies and the chiral magnetic effect in Fermi liquids, Phys. Rev. Lett. 109 (2012) 181602 [arXiv:1203.2697] [INSPIRE].

[61] K. Fujikawa, Characteristics of chiral anomaly in view of various applications, Phys. Rev. D 97 (2018) 016018 [arXiv:1709.08181] [INSPIRE].

[62] K.-I. Shizuya, Conformal anomalies in quantum chromodynamics and the path integral method, Phys. Rev. D 35 (1987) 2550 [InSPIRE].

[63] S.D. Joglekar and A. Misra, Observations on path integral derivations of anomalies, Phys. Rev. D 36 (1987) 642 [INSPIRE].

[64] J. Manes, R. Stora and B. Zumino, Algebraic study of chiral anomalies, Commun. Math. Phys. 102 (1985) 157 [INSPIRE].

[65] M. Dubois-Violette, M. Henneaux, M. Talon and C.-M. Viallet, Some results on local cohomologies in field theory, Phys. Lett. B 267 (1991) 81 [InSPIRE].

[66] L. Baulieu and J. Thierry-Mieg, Algebraic structure of quantum gravity and the classification of the gravitational anomalies, Phys. Lett. B 145 (1984) 53 [INSPIRE].

[67] L. Bonora, P. Pasti and M. Tonin, The anomaly structure of theories with external gravity, J. Math. Phys. 27 (1986) 2259 [INSPIRE].

[68] S.P. Sorella, Algebraic characterization of the Wess-Zumino consistency conditions in gauge theories, Commun. Math. Phys. 157 (1993) 231 [hep-th/9302136] [INSPIRE].

[69] C. Becchi, A. Rouet and R. Stora, Renormalization of the Abelian Higgs-Kibble model, Commun. Math. Phys. 42 (1975) 127 [INSPIRE].

[70] S.L. Adler and W.A. Bardeen, Absence of higher order corrections in the anomalous axial vector divergence equation, Phys. Rev. 182 (1969) 1517 [INSPIRE].

[71] W.A. Bardeen, Anomalous Ward identities in spinor field theories, Phys. Rev. 184 (1969) 1848 [INSPIRE].

[72] B. Zumino, Y.-S. Wu and A. Zee, Chiral anomalies, higher dimensions and differential geometry, Nucl. Phys. B 239 (1984) 477 [INSPIRE].

[73] W.A. Bardeen and B. Zumino, Consistent and covariant anomalies in gauge and gravitational theories, Nucl. Phys. B 244 (1984) 421 [INSPIRE].

[74] T. O'Brien, C. Beenakker and I. Adagideli, Superconductivity provides access to the chiral magnetic effect of an unpaired Weyl cone, Phys. Rev. Lett. 118 (2017) 207701 [arXiv: 1612.06848] [INSPIRE].

[75] L.D. Landau and E.M. Lifschitz, Quantum mechanics, Pergamon Press, U.K., (1965).

[76] N.K. Nielsen and B. Schroer, Axial anomaly and Atiyah-Singer theorem, Nucl. Phys. B 127 (1977) 493 [inSPIRE].

[77] C. Callias, Index theorems on open spaces, Commun. Math. Phys. 62 (1978) 213 [INSPIRE].

[78] L. Álvarez-Gaumé, A note on the Atiyah-Singer index theorem, J. Phys. A 16 (1983) 4177 [INSPIRE]. 
[79] D. Friedan and P. Windey, Supersymmetric derivation of the Atiyah-Singer index and the chiral anomaly, Nucl. Phys. B 235 (1984) 395 [INSPIRE].

[80] K. Symanzik, Euclidean quantum field theory. I. Equations for a scalar model, J. Math. Phys. 7 (1966) 510.

[81] H.M. Price, O. Zilberberg, T. Ozawa, I. Carusotto and N. Goldman, Four-dimensional quantum Hall effect with ultracold atoms, Phys. Rev. Lett. 115 (2015) 195303 [arXiv: 1505.04387] [INSPIRE].

[82] M. Lohse, C. Schweizer, H.M. Price, O. Zilberberg and I. Bloch, Exploring $4 D$ quantum Hall physics with a $2 D$ topological charge pump, Nature 553 (2018) 55.

[83] M. Ezawa, Chiral anomaly enhancement and photoirradiation effects in multiband touching fermion systems, Phys. Rev. B 95 (2017) 205201 [arXiv:1612.05857] [INSPIRE].

[84] A.A. Zyuzin, S. Wu and A.A. Burkov, Weyl semimetal with broken time reversal and inversion symmetries, Phys. Rev. B 85 (2012) 165110 [arXiv:1201.3624] [INSPIRE].

[85] W.A. Bardeen and B. Zumino, Consistent and covariant anomalies in gauge and gravitational theories, Nucl. Phys. B 244 (1984) 421 [INSPIRE].

[86] A.A. Belavin, A.M. Polyakov, A.S. Schwartz and Yu. S. Tyupkin, Pseudoparticle solutions of the Yang-Mills equations, Phys. Lett. B 59 (1975) 85 [InSPIRE].

[87] A.M. Polyakov, Quark confinement and topology of gauge groups, Nucl. Phys. B 120 (1977) 429 [INSPIRE].

[88] G. 't Hooft, Symmetry breaking through Bell-Jackiw anomalies, Phys. Rev. Lett. 37 (1976) 8 [INSPIRE].

[89] G. 't Hooft, Computation of the quantum effects due to a four-dimensional pseudoparticle, Phys. Rev. D 14 (1976) 3432 [Erratum ibid. D 18 (1978) 2199] [InSPIRE].

[90] S.R. Coleman, J. Wess and B. Zumino, Structure of phenomenological Lagrangians. 1, Phys. Rev. 177 (1969) 2239 [inSPIRE].

[91] C.G. Callan Jr., S.R. Coleman, J. Wess and B. Zumino, Structure of phenomenological Lagrangians. 2, Phys. Rev. 177 (1969) 2247 [INSPIRE].

[92] S.P. Novikov, The Hamiltonian formalism and a many valued analog of Morse theory, Usp. Mat. Nauk 37N5 (1982) 3 [Russ. Math. Surveys 37 (1982) 1] [INSPIRE].

[93] E. Witten, Global aspects of current algebra, Nucl. Phys. B 223 (1983) 422 [InSPIRE].

[94] C.G. Callan Jr., R.F. Dashen and D.J. Gross, The structure of the gauge theory vacuum, Phys. Lett. B 63 (1976) 334 [InSPIRE].

[95] R. Jackiw and C. Rebbi, Vacuum periodicity in a Yang-Mills quantum theory, Phys. Rev. Lett. 37 (1976) 172 [INSPIRE].

[96] B. Holdom, A realistic model with dynamically broken symmetries, Phys. Rev. D 23 (1981) 1637 [INSPIRE].

[97] S. Weinberg, Color and electroweak forces as a source of quark and lepton masses, Phys. Lett. B 102 (1981) 401 [INSPIRE].

[98] E. Guadagnini and K. Konishi, Composite quarks and Cabibbo mixing, Nucl. Phys. B 196 (1982) 165 [INSPIRE]. 
[99] V.A. Rubakov, Adler-Bell-Jackiw anomaly and fermion number breaking in the presence of a magnetic monopole, Nucl. Phys. B 203 (1982) 311 [INSPIRE].

[100] F. Wilczek, Magnetic flux, angular momentum and statistics, Phys. Rev. Lett. 48 (1982) 1144 [INSPIRE].

[101] E. Guadagnini, Baryons as solitons and mass formulae, Nucl. Phys. B 236 (1984) 35 [INSPIRE].

[102] R.J. Crewther, Effects of topological charge in gauge theories, in Facts and prospects of gauge theories, Acta Phys. Austriaca Suppl. 19 (1978) 47 [INSPIRE].

[103] A.A. Soluyanov et al., Type-II Weyl semimetals, Nature 527 (2015) 495.

[104] P. Kim, J.H. Ryoo and C.-H. Park, Breakdown of the chiral anomaly in Weyl semimetals in a strong magnetic field, Phys. Rev. Lett. 119 (2017) 266401 [arXiv:1707.01103] [INSPIRE]. 